\title{
Derivation of the Landau-Pekar Equations in a Many-Body Mean-Field Limit
}

\author{
Nikolai Leopold®, David Mitrouskas \& Robert Seiringer
}

\author{
Communicated by C. Mouнот
}

\begin{abstract}
We consider the Fröhlich Hamiltonian in a mean-field limit where many bosonic particles weakly couple to the quantized phonon field. For large particle numbers and a suitably small coupling, we show that the dynamics of the system is approximately described by the Landau-Pekar equations. These describe a Bose-Einstein condensate interacting with a classical polarization field, whose dynamics is effected by the condensate, i.e., the back-reaction of the phonons that are created by the particles during the time evolution is of leading order.
\end{abstract}

\section{Introduction}

We consider the dynamics of $N$ bosonic particles interacting with a quantized phonon field described by the Fröhlich model in a mean field regime. The underlying Hilbert space is

$$
\mathcal{H}^{(N)}=L_{s}^{2}\left(\mathbb{R}^{3 N}\right) \otimes \mathcal{F}_{s},
$$

where the $N$ particles are described by states in $L_{s}^{2}\left(\mathbb{R}^{3 N}\right)$, the subspace of all complex-valued square integrable $N$-particle wave functions that are symmetric under the exchange of any pair of the coordinates $\left(x_{1}, \ldots, x_{N}\right)$, and where the phonon field is represented by elements in the bosonic Fock space $\mathcal{F}_{s}=\bigoplus_{n \geqq 0} L_{s}^{2}\left(\mathbb{R}^{3 n}\right)$. The time evolution of the system is governed by the Schrödinger equation

$$
i \partial_{t} \Psi_{N, t}=H_{N, \alpha}^{\mathrm{F}} \Psi_{N, t},
$$

with the Fröhlich Hamiltonian

$$
H_{N, \alpha}^{\mathrm{F}}=\sum_{j=1}^{N}\left[-\Delta_{j}+\sqrt{\frac{\alpha}{N}} \int d^{3} k|k|^{-1}\left(e^{i k x_{j}} a_{k}+e^{-i k x_{j}} a_{k}^{*}\right)\right]+\mathcal{N} .
$$


Here, $\Delta_{j}$ is the Laplacian acting on the $j$ th particle with coordinate $x_{j}, a_{k}$ and $a_{k}^{*}$ denote the usual bosonic annihilation and creation operators satisfying the canonical commutation relations

$$
\left[a_{k}, a_{l}^{*}\right]=\delta(k-l), \quad\left[a_{k}, a_{l}\right]=\left[a_{k}^{*}, a_{l}^{*}\right]=0,
$$

and $\mathcal{N}$ is the number operator defined by $\mathcal{N}=\int d^{3} k a_{k}^{*} a_{k}$. The coupling parameter $\sqrt{\alpha / N}$ is introduced to scale the strength of the interaction between the particles and the phonon field. If the number of phonons is of order $N$ and $\alpha>0$ is fixed, the factor $N^{-1 / 2}$ and the fact that the creation and annihilation operators scale like $\sqrt{N}$ (they are bounded by $(\mathcal{N}+1)^{1 / 2}$, see (3.3)) ensure that the kinetic and potential energy are of the same order for large $N$.

We note that the expression (1.3) is somewhat formal, since the form factor $|k|^{-1}$ in the interaction term is not square integrable. By a well-known argument going back to Lieb and Yamazaki [26] (cf. Lemma A.1), the right side of (1.3) defines a closed bounded from below quadratic form with domain given by the form domain of $H_{N, 0}^{\mathrm{F}}$. The self-adjoint operator that corresponds to this form is called Fröhlich Hamiltonian and denoted by $H_{N, \alpha}^{\mathrm{F}}$. We refer to [16] for a detailed description of its domain $\mathcal{D}\left(H_{N, \alpha}^{\mathrm{F}}\right)$ (see also Lemma 3.1).

If the number $N$ of particles is large, we show for a particular class of initial states that the solution of the many-body Schrödinger equation (1.2) can be approximated by Pekar product states, i.e., states of the form

$$
\Psi_{N, t}=\psi_{t}^{\otimes N} \otimes W\left(\sqrt{N} \varphi_{t}\right) \Omega
$$

where $\Omega$ is the vacuum state in $\mathcal{F}_{s}, W$ denotes the Weyl operator and $\left(\psi_{t}, \varphi_{t}\right) \in$ $L^{2}\left(\mathbb{R}^{3}\right) \times L^{2}\left(\mathbb{R}^{3}\right)$ solve the time-dependent Landau-Pekar equations

$$
\left\{\begin{array}{l}
i \partial_{t} \psi_{t}(x)=\left[-\Delta_{x}+\sqrt{\alpha} \Phi(x, t)\right] \psi_{t}(x) \\
i \partial_{t} \varphi_{t}(k)=\varphi_{t}(k)+\sqrt{\alpha}|k|^{-1} \int d^{3} x e^{-i k x}\left|\psi_{t}(x)\right|^{2}
\end{array}\right.
$$

where

$$
\Phi(x, t)=\int d^{3} k|k|^{-1}\left(e^{i k x} \varphi_{t}(k)+e^{-i k x} \overline{\varphi_{t}(k)}\right) .
$$

The Weyl operator is defined for any $f \in L^{2}\left(\mathbb{R}^{3}\right)$ by

$$
W(f)=\exp \left(\int d^{3} k\left(f(k) a_{k}^{*}-\overline{f(k)} a_{k}\right)\right) .
$$

In the Pekar product state (1.5), the phonons are in the coherent state $W\left(\sqrt{N} \varphi_{t}\right) \Omega$ with average number of excitations of order $N$, and the bosonic particles form a pure Bose-Einstein condensate with condensate wave function $\psi_{t}$. According to the Landau-Pekar equations, the one-particle condensate wave function $\psi_{t}$ evolves in the potential $\sqrt{\alpha} \Phi(x, t)$ created by the phonons, while the phonon field couples to the particles via the source term involving the density $\left|\psi_{t}(x)\right|^{2}$. 
Our main result can be summarized as follows: given an initial wave function $\Psi_{N, 0}$ that is close to a Pekar product state $\psi_{0}^{\otimes N} \otimes W\left(\sqrt{N} \varphi_{0}\right) \Omega$ (close in an appropriate sense that will be specified in the next section), then the time evolved state $e^{-i H_{N, \alpha}^{\mathrm{F}} t} \Psi_{N, 0}$ remains close to the time evolved Pekar state (1.5) when $N \gg 1$.

The Landau-Pekar equations were originally introduced in [21] to approximate the time evolution of a single polaron in the strong coupling limit. In our notation, the strong coupling regime corresponds to the Hamiltonian $H_{1, \alpha}^{\mathrm{F}}$ with $\alpha \gg 1$. Partial results concerning a rigorous derivation of the Landau-Pekar equations in the strong coupling limit were obtained in $[8,10,15,25]$ (for a detailed comparison between the different results we refer to [25, Chapter 2]). In these works, the Landau-Pekar equations are justified for short times, namely at most for times of order $\alpha^{-\varepsilon}$ with $\varepsilon>0$ arbitrary small. ${ }^{1}$ A derivation for times of order one, the time scale in the strong coupling limit at which the back-reaction of the phonons that are created during the time evolution is of leading order, remains an open problem. The emergence of classical radiation in the strong coupling limit is expected to rely on the adiabatic decoupling between the relatively fast moving (with respect to $\alpha$ ) electron and the radiation field. For results on adiabatic theorems of the LandauPekar equations in one and three dimensions we refer to $[9,25]$.

In the many-particle mean-field limit considered in this work, the creation of coherent radiation happens for a different reason than in the strong coupling regime, namely because there are many particles in the same quantum state that simultaneously create the phonons. In this regard, the present work is related to [1,7,22-24], where many-body mean-field limits of the renormalized Nelson model, the Nelson model with ultraviolet cutoff and the (bosonic) Pauli-Fierz model are considered. In particular, we mention [1] where the Schrödinger-Klein-Gordon equations were derived by the Wigner measure approach as a limit of the renormalized Nelson model.

In $[4,5,12]$, effective equations for the Nelson, Pauli-Fierz and Fröhlich model were derived in a partially classical limit. There, the number of particles is kept fixed while the number of excitations of the quantum field tends to infinity and the coupling constant approaches zero in a suitable sense. The effect of the excitations that are created during time evolution is negligible in this limit and the quantum field can thus be approximated by a classical field that evolves freely or remains constant in time.

To the best of our knowledge, the present work provides the first derivation of the Landau-Pekar equations in a limit in which the back-reaction of the phonons that are created during time evolution is of leading order. Moreover, our results include explicit error estimates.

In order to derive our results, we follow [24], which combines the methods from [29,31]. The new technical challenge in comparison with [24] is to show that the high momentum phonons do not obstruct the expected mean-field behavior.

${ }^{1}$ It should be noted that results about the polaron in the strong coupling limit are usually formulated in strong coupling units and that times of order $\alpha^{2}$ in the stated references correspond to times of order one in the units of the present paper. 
This requires several nontrivial modifications. First, it is crucial to introduce a measure for the excitations around the condensate resp. around the coherent state that involves the canonical transformation due to Gross and Nelson (see (2.12)). In particular, we use the representation of the Fröhlich Hamiltonian in [16]. The most difficult part is to control the interaction between the ultraviolet modes of the phonon field and the fraction of particles not in the condensate. To this end, we restrict our consideration to a subclass of the initial states which have small fluctuations in the energy per particle observable and combine estimates similar to [23, Sect. VIII.1] with an operator bound that is motivated by [10, Lemma 10]. The idea of using this restriction in order to treat the singular interaction between quantum fields and particles in the mean field regime was already used in [23].

The article is organized as follows: in the next section, we state our main results. In Theorem 2.1, we consider initial states in the domain of the Fröhlich Hamiltonian, while Theorem 2.2 is about initial states in the domain of the noninteracting model (including, in particular, product states). In Section 3, we introduce useful notation and discuss the representation of the Fröhlich Hamiltonian via the Gross transformation. The key steps of the proof of our main result are summarized in Section 4 in terms of several lemmas. The proofs of these are given in Sections 5-7.

\section{Main Results}

For notational convenience, we set the coupling constant $\alpha=1$ from now on and denote $H_{N}^{\mathrm{F}}=H_{N, 1}^{\mathrm{F}}$. All statements and proofs that follow are, however, equally true for any $\alpha>0$ independent of $N$.

In order to state our main results we define for $\Psi_{N} \in \mathcal{H}^{(N)}$ the one-particle reduced density matrix

$$
\gamma_{\Psi_{N}}^{(1,0)}=\operatorname{Tr}_{2, \ldots, N} \otimes \operatorname{Tr}_{\mathcal{F}_{S}}\left|\Psi_{N}\right\rangle\left\langle\Psi_{N}\right|
$$

on the Hilbert space $L^{2}\left(\mathbb{R}^{3}\right)$. Here, $\operatorname{Tr}_{2, \ldots, N}$ denotes the partial trace over the coordinates $x_{2}, \ldots, x_{N}$ and $\operatorname{Tr}_{\mathcal{F}_{s}}$ the trace over Fock space. The particles of a many-body state $\Psi_{N}$ are said to exhibit complete Bose-Einstein condensation if there exists $\psi \in L^{2}\left(\mathbb{R}^{3}\right)$ with $\|\psi\|_{L^{2}\left(\mathbb{R}^{3}\right)}=1$ such that

$$
\left.\operatorname{Tr}_{L^{2}\left(\mathbb{R}^{3}\right)}\left|\gamma_{\Psi_{N}}^{(1,0)}-\right| \psi\right\rangle\langle\psi|| \rightarrow 0
$$

as $N \rightarrow \infty$. In this case $\psi$ is called the condensate wave function. Moreover, we define (for $(\psi, \varphi) \in L^{2}\left(\mathbb{R}^{3}\right) \times L^{2}\left(\mathbb{R}^{3}\right)$ and $\Psi_{N} \in \mathcal{D}\left(H_{N}^{\mathrm{F}}\right)$ )

$$
\begin{aligned}
a\left(\Psi_{N}, \psi\right) & \left.=\operatorname{Tr}_{L^{2}\left(\mathbb{R}^{3}\right)}\left|\gamma_{\Psi_{N}}^{(1,0)}-\right| \psi\right\rangle\langle\psi||, \\
b\left(\Psi_{N}, \varphi\right) & =N^{-1}\left\langle W^{*}(\sqrt{N} \varphi) \Psi_{N}, \mathcal{N} W^{*}(\sqrt{N} \varphi) \Psi_{N}\right\rangle, \\
c\left(\Psi_{N}\right) & =\left\|N^{-1}\left(H_{N}^{\mathrm{F}}-\left\langle\Psi_{N}, H_{N}^{\mathrm{F}} \Psi_{N}\right\rangle\right) \Psi_{N}\right\|^{2} .
\end{aligned}
$$

For $m \in \mathbb{N}$, let $H^{m}\left(\mathbb{R}^{3}\right)$ denote the Sobolev space of order $m$ and $L_{m}^{2}\left(\mathbb{R}^{3}\right)$ a weighted $L^{2}$-space with norm $\|\varphi\|_{L_{m}^{2}\left(\mathbb{R}^{3}\right)}=\left\|\left(1+|\cdot|^{2}\right)^{m / 2} \varphi\right\|_{L^{2}\left(\mathbb{R}^{3}\right)}$. We will use the following result which was proven in [8]: 
Proposition 2.1. (Lemma C.2 in [8]) The Landau-Pekar equations (1.6) are globally well-posed in $H^{2}\left(\mathbb{R}^{3}\right) \times L_{1}^{2}\left(\mathbb{R}^{3}\right)$. For all $t \in \mathbb{R}$ we have

$$
\left\|\psi_{t}\right\|_{H^{2}\left(\mathbb{R}^{3}\right)} \leqq C(1+|t|) \quad \text { and } \quad\left\|\varphi_{t}\right\|_{L_{1}^{2}\left(\mathbb{R}^{3}\right)} \leqq C(1+|t|),
$$

where $C$ is a constant depending only on the initial data.

We are now ready to state our main theorem.

Theorem 2.1. Let $(\psi, \varphi) \in H^{2}\left(\mathbb{R}^{3}\right) \times L_{1}^{2}\left(\mathbb{R}^{3}\right)$ s.t. $\|\psi\|_{L^{2}\left(\mathbb{R}^{3}\right)}=1$, and $\Psi_{N} \in$ $\mathcal{D}\left(H_{N}^{\mathrm{F}}\right)$ s.t. $\left\|\Psi_{N}\right\|=1$ and $E_{0}=\sup _{N \in \mathbb{N}}\left|N^{-1}\left\langle\Psi_{N}, H_{N}^{\mathrm{F}} \Psi_{N}\right\rangle\right|<\infty$. Let $\left(\psi_{t}, \varphi_{t}\right)$ be the unique solution of (1.6) with initial datum $(\psi, \varphi)$ and $\Psi_{N, t}=e^{-i H_{N}^{\mathrm{F}} t} \Psi_{N}$. Then, there exists a constant $C>0$ (depending only on $\|\varphi\|_{L_{1}^{2}\left(\mathbb{R}^{3}\right)},\|\psi\|_{H^{2}\left(\mathbb{R}^{3}\right)}$ and $\left.E_{0}\right)$ such that

$$
\begin{aligned}
\left.\operatorname{Tr}_{L^{2}\left(\mathbb{R}^{3}\right)}\left|\gamma_{\Psi_{N, t}}^{(1,0)}-\right| \psi_{t}\right\rangle\left\langle\psi_{t}||\right. & \leqq \sqrt{a\left(\Psi_{N}, \psi\right)+b\left(\Psi_{N}, \varphi\right)+c\left(\Psi_{N}\right)+N^{-1 / 2}} e^{C(1+|t|)^{3}} \\
N^{-1}\left\langle W^{*}\left(\sqrt{N} \varphi_{t}\right) \Psi_{N, t},\right. & \left.\mathcal{N} W^{*}\left(\sqrt{N} \varphi_{t}\right) \Psi_{N, t}\right\rangle \\
& \leqq\left(a\left(\Psi_{N}, \psi\right)+b\left(\Psi_{N}, \varphi\right)+c\left(\Psi_{N}\right)+N^{-1 / 2}\right) e^{C(1+|t|)^{3}} .
\end{aligned}
$$

The proof is given in Section 4.

Remark 2.1. If one considers initial many-body states in which the particles are in a Bose-Einstein condensate, the phonons are in a coherent states and the energy has small fluctuations around its mean value, i.e.

$$
\lim _{N \rightarrow \infty}\left(a\left(\Psi_{N}, \psi\right)+b\left(\Psi_{N}, \varphi\right)+c\left(\Psi_{N}\right)\right)=0,
$$

it follows from Theorem 2.1 that

$$
\begin{aligned}
\left.\lim _{N \rightarrow \infty} \operatorname{Tr}_{L^{2}\left(\mathbb{R}^{3}\right)}\left|\gamma_{\Psi_{N, t}}^{(1,0)}-\right| \psi_{t}\right\rangle\left\langle\psi_{t}||\right. & =0 \text { and } \\
\lim _{N \rightarrow \infty} N^{-1}\left\langle W^{*}\left(\sqrt{N} \varphi_{t}\right) \Psi_{N, t}, \mathcal{N} W^{*}\left(\sqrt{N} \varphi_{t}\right) \Psi_{N, t}\right\rangle & =0 .
\end{aligned}
$$

Our result consequently shows the stability of the condensate and the coherent state during the time evolution.

Remark 2.2. The condition $c\left(\Psi_{N}\right) \rightarrow 0$ as $N \rightarrow \infty$ restricts the initial data to many-body states $\Psi_{N}$ whose energy per particle has small fluctuations around its mean value. In our proof, this is important to obtain sufficient control on the singular ultraviolet behavior of the interaction term in $H_{N}^{\mathrm{F}}$. We give a detailed explanation of this point in Section 5. In the presence of an ultraviolet cutoff in the Fröhlich Hamiltonian, the estimates (2.7) and (2.8) hold without the appearance of $c\left(\Psi_{N}\right)$ on the right hand side, but with a cutoff dependent constant $C$. In this simpler case, the statement could be proven in close analogy to $[7,24]$ where the Nelson model was considered with ultraviolet cutoff. 
Next, we give examples of initial states that satisfy (2.9). The quantities $a\left(\Psi_{N}, \psi\right)$ and $b\left(\Psi_{N}, \varphi\right)$ are identically zero for Pekar product states $\Psi_{N}=\psi^{\otimes N} \otimes W(\sqrt{N} \varphi) \Omega$ with $(\psi, \varphi) \in H^{2}\left(\mathbb{R}^{3}\right) \times L_{1}^{2}\left(\mathbb{R}^{3}\right)$. However, such Pekar states are in the domain $\mathcal{D}\left(H_{N}^{0}\right)=\left(H_{s}^{2}\left(\mathbb{R}^{3 N}\right) \otimes \mathcal{F}_{s}\right) \cap \mathcal{D}(\mathcal{N})$ of the free Hamiltonian

$$
H_{N}^{0}=-\sum_{j=1}^{N} \Delta_{j}+\mathcal{N}
$$

and thus, as shown in [16], can not be elements of $\mathcal{D}\left(H_{N}^{\mathrm{F}}\right)$. As a consequence, $c\left(\Psi_{N}\right)$ would be infinite in this case. To specify states that satisfy (2.9), we introduce the Gross transform

$$
U_{K}=\exp \left[N^{-1 / 2} \sum_{j=1}^{N} \int d^{3} k\left(\overline{B_{K, x_{j}}(k)} a_{k}-B_{K, x_{j}}(k) a_{k}^{*}\right)\right]
$$

where

$$
B_{K, x}(k)=\frac{-1}{|k|\left(1+k^{2}\right)} e^{-i k x} \mathbb{1}_{|k| \geqq K}(k)
$$

for $0<K<\infty$. The Gross transform, which goes back to Gross and Nelson [17,28], relates the domains of $H_{N}^{0}$ and $H_{N}^{\mathrm{F}}$ to each other. ${ }^{2}$ In Lemma 3.1 we show that there is a $\widetilde{K}>0$ such that for all $K \geqq \widetilde{K}$ and all $N \geqq 1$, the domains satisfy

$$
\mathcal{D}\left(H_{N}^{\mathrm{F}}\right)=U_{K}^{*} \mathcal{D}\left(H_{N}^{0}\right)
$$

If we choose $K$ as an $N$-dependent sufficiently rapidly growing sequence $\left(K_{N}\right)_{N \geqq 1}$, then the Gross transform $U_{K_{N}}$ has negligible effect on the condensate and the coherent state structure. This is summarized in the next proposition.

Proposition 2.2. Assume $K \geqq c$ for some $c>0$ and consider the state $\Psi_{N}=$ $U_{K}^{*}\left(\psi^{\otimes N} \otimes W(\sqrt{N} \varphi) \Omega\right)$ with $(\psi, \varphi) \in H^{2}\left(\mathbb{R}^{3}\right) \times L_{1}^{2}\left(\mathbb{R}^{3}\right)$ and $\|\psi\|_{L^{2}\left(\mathbb{R}^{3}\right)}=1$. Then there exists a $C>0$ such that $\sup _{N \in \mathbb{N}}\left|N^{-1}\left\langle\Psi_{N}, H_{N}^{\mathrm{F}} \Psi_{N}\right\rangle\right| \leqq C$ and

$$
a\left(\Psi_{N}, \psi\right) \leqq \frac{C}{K^{3 / 2}}, \quad b\left(\Psi_{N}, \varphi\right) \leqq \frac{C}{K^{3}}, \quad c\left(\Psi_{N}\right) \leqq C\left(K^{-1}+N^{-1}+\frac{K}{N^{2}}\right)
$$

with $a\left(\Psi_{N}, \psi\right), b\left(\Psi_{N}, \varphi\right)$ and $c\left(\Psi_{N}\right)$ defined as in Theorem 2.1.

We prove this proposition in Section 7.2. As an immediate consequence of Proposition 2.2 (with $K=c N$ ) and Theorem 2.1 one finds

$$
\left.\operatorname{Tr}_{L^{2}\left(\mathbb{R}^{3}\right)}\left|\gamma_{\Psi_{N, t}}^{(1,0)}-\right| \psi_{t}\right\rangle\left\langle\psi_{t}|| \leqq N^{-1 / 4} e^{C(1+|t|)^{3}}\right.
$$

2 The Gross transform adds correlations between the bosons and phonon modes with momentum $|k| \geq K$. This leads to a better ultraviolet behavior of the radiation field. 
and

$$
\frac{1}{N}\left\langle W^{*}\left(\sqrt{N} \varphi_{t}\right) \Psi_{N, t}, \mathcal{N} W^{*}\left(\sqrt{N} \varphi_{t}\right) \Psi_{N, t}\right\rangle \leqq N^{-1 / 2} e^{C(1+|t|)^{3}}
$$

for initial states of the form $\Psi_{N}=U_{c N}^{*}\left(\psi^{\otimes N} \otimes W(\sqrt{N} \varphi) \Omega\right)$.

Since the quantities $b\left(\Psi_{N}, \varphi\right)$ and $c\left(\Psi_{N}\right)$ appearing on the right side of (2.7) and (2.8) are expectation values of unbounded operators, it is not possible to generalize Theorem 2.1 to initial states $\Psi_{N} \notin \mathcal{D}\left(H_{N}^{\mathrm{F}}\right)$ via a simple density argument. Using the Gross transform, however, it is possible to obtain a similar result for initial states in a subset of $\mathcal{D}\left(H_{N}^{0}\right)$. This follows from Theorem 2.1 in combination with (2.14) and the fact that $U_{K}$ converges strongly to the identity operator for $K \rightarrow \infty$. The precise statement is as follows:

Theorem 2.2. Let $K_{N} \geqq c N^{5 / 6}$ for some $c>0$. Let $(\psi, \varphi) \in H^{2}\left(\mathbb{R}^{3}\right) \times L_{1}^{2}\left(\mathbb{R}^{3}\right)$ with $\|\psi\|_{L^{2}\left(\mathbb{R}^{3}\right)}=1$, and $\Psi_{N} \in \mathcal{D}\left(H_{N}^{0}\right)$ such that $\left\|\Psi_{N}\right\|=1$ and

$$
E_{0}=\sup _{N \in \mathbb{N}}\left|N^{-1}\left\langle\Psi_{N}, U_{K_{N}} H_{N}^{\mathrm{F}} U_{K_{N}}^{*} \Psi_{N}\right\rangle\right|<\infty
$$

Let $\left(\psi_{t}, \varphi_{t}\right)$ be the unique solution of (1.6) with initial datum $(\psi, \varphi)$ and $\Psi_{N, t}=$ $e^{-i H_{N}^{\mathrm{F}} t} \Psi_{N}$. Then, there exists a constant $C>0$ (depending only on $c,\|\varphi\|_{L_{1}^{2}\left(\mathbb{R}^{3}\right)}$, $\|\psi\|_{H^{2}\left(\mathbb{R}^{3}\right)}$ and $\left.E_{0}\right)$ such that

$$
\begin{aligned}
& \left.\operatorname{Tr}_{L^{2}\left(\mathbb{R}^{3}\right)}\left|\gamma_{\Psi_{N, t}}^{(1,0)}-\right| \psi_{t}\right\rangle\left\langle\psi_{t}|| \leqq \sqrt{a\left(\Psi_{N}, \psi\right)+b\left(\Psi_{N}, \varphi\right)+c\left(U_{K_{N}}^{*} \Psi_{N}\right)+N^{-1 / 2}} e^{C(1+|t|)^{3}},\right. \\
& \left\langle W^{*}\left(\sqrt{N} \varphi_{t}\right) \Psi_{N, t}, \sqrt{\frac{\mathcal{N}}{N}} W^{*}\left(\sqrt{N} \varphi_{t}\right) \Psi_{N, t}\right\rangle \\
& \leqq \sqrt{a\left(\Psi_{N}, \psi\right)+b\left(\Psi_{N}, \varphi\right)+c\left(U_{K_{N}}^{*} \Psi_{N}\right)+N^{-1 / 2}} e^{C(1+|t|)^{3}}
\end{aligned}
$$

In particular, for the Pekar initial state $\Psi_{N}=\psi^{\otimes N} \otimes W(\sqrt{N} \varphi) \Omega$ we have the bounds

$$
\begin{aligned}
& \left.\operatorname{Tr}_{L^{2}\left(\mathbb{R}^{3}\right)}\left|\gamma_{\Psi_{N, t}}^{(1,0)}-\right| \psi_{t}\right\rangle\left\langle\psi_{t}|| \leqq N^{-1 / 4} e^{C(1+|t|)^{3}}\right. \\
& \left\langle W^{*}\left(\sqrt{N} \varphi_{t}\right) \Psi_{N, t}, \sqrt{\frac{\mathcal{N}}{N}} W^{*}\left(\sqrt{N} \varphi_{t}\right) \Psi_{N, t}\right\rangle \leqq N^{-1 / 4} e^{C(1+|t|)^{3}} .
\end{aligned}
$$

The proof is given in Section 7.3.

Remark 2.3. The restriction $K_{N} \geqq c N^{5 / 6}$ was chosen in order to minimize the error terms in (2.19) and (2.20).

Remark 2.4. Note that in (2.20) we only control the time evolution of $\sqrt{N^{-1} \mathcal{N}}$, while in (2.8) we estimate the operator $N^{-1} \mathcal{N}$. 


\section{Preliminaries}

\subsection{Notation and Basic Estimates}

We introduce the usual bosonic creation and annihilation operators

$$
a(f)=\int d^{3} k \overline{f(k)} a_{k}, \quad a^{*}(f)=\int d^{3} k f(k) a_{k}^{*}, \quad f \in L^{2}\left(\mathbb{R}^{3}\right),
$$

as well as the field operators

$$
\Phi(f)=a(f)+a^{*}(f), \quad \Pi(f)=\Phi(i f)=i\left(-a(f)+a^{*}(f)\right) .
$$

They satisfy the bounds

$$
\left\|a(f) \Psi_{N}\right\| \leqq\|f\|_{L^{2}\left(\mathbb{R}^{3}\right)}\left\|\mathcal{N}^{1 / 2} \Psi_{N}\right\|, \quad\left\|a^{*}(f) \Psi_{N}\right\| \leqq\|f\|_{L^{2}\left(\mathbb{R}^{3}\right)}\left\|(\mathcal{N}+1)^{1 / 2} \Psi_{N}\right\|,
$$

and

$$
\begin{aligned}
& \left\|\Phi(f) \Psi_{N}\right\| \leqq 2\|f\|_{L^{2}\left(\mathbb{R}^{3}\right)}\left\|(\mathcal{N}+1)^{1 / 2} \Psi_{N}\right\|, \\
& \left\|\Pi(f) \Psi_{N}\right\| \leqq 2\|f\|_{L^{2}\left(\mathbb{R}^{3}\right)}\left\|(\mathcal{N}+1)^{1 / 2} \Psi_{N}\right\|
\end{aligned}
$$

for any $\Psi_{N} \in \mathcal{H}^{(N)}$. For $K>0$, we define the classical fields

$$
\begin{gathered}
\Phi_{K}(x, t)=\int_{|k| \leqq K} d^{3} k|k|^{-1}\left(e^{i k x} \varphi_{t}(k)+e^{-i k x} \overline{\varphi_{t}(k)}\right), \\
\Phi_{\geqq K}(x, t)=\int_{|k| \geqq K} d^{3} k|k|^{-1}\left(e^{i k x} \varphi_{t}(k)+e^{-i k x} \overline{\varphi_{t}(k)}\right) .
\end{gathered}
$$

Moreover, it is useful to define the functions

$$
G_{x}(k)=e^{-i k x}|k|^{-1}, \quad G_{K, x}(k)=e^{-i k x}|k|^{-1} \mathbb{1}_{|k| \leqq K}(k),
$$

and $B_{K, x}(k)=\frac{-1}{|k|\left(1+k^{2}\right)} e^{-i k x} \mathbb{1}_{|k| \geqq K}(k)$ as in (2.13). The bounds

$$
\left\|G_{K, x}\right\|_{L^{2}\left(\mathbb{R}^{3}\right)}^{2}=4 \pi K, \quad\left\|B_{K, x}\right\|_{L^{2}\left(\mathbb{R}^{3}\right)}^{2} \leqq 4 \pi K^{-3}, \quad\left\||\cdot| B_{K, x}\right\|_{L^{2}\left(\mathbb{R}^{3}\right)}^{2} \leqq 4 \pi K^{-1}
$$

are straightforward to verify and will be frequently used in the rest of the article. We also have

$$
\left|\Phi_{K}(x, t)\right| \leqq \sqrt{32 \pi}\left\|\varphi_{t}\right\|_{L_{1}^{2}\left(\mathbb{R}^{3}\right)}, \quad\left\|\Phi\left(G_{K, x_{j}}\right) \Psi_{N}\right\| \leqq \sqrt{16 \pi K}\left\|(\mathcal{N}+1)^{1 / 2} \Psi_{N}\right\|
$$

for $j \in\{1, \ldots, N\}$.

Notation: The functions $k \mapsto k G_{K, x}(k)$ and $k \mapsto k B_{K, x}(k)$ will frequently be denoted by $k G_{K, x}$ and $k B_{K, x}$, respectively. Depending on the context $\|\cdot\|$ and $\langle\cdot, \cdot\rangle$ will refer to the norm and scalar product either of $\mathcal{H}^{(N)}$ or $L^{2}\left(\mathbb{R}^{3}\right)$. If the spaces $L_{m}^{2}\left(\mathbb{R}^{3}\right)$ and $H^{m}\left(\mathbb{R}^{3}\right)$ (with $m \in \mathbb{N}$ ) appear as subscripts we will abbreviate them by $L_{m}^{2}$ and $H^{m}$. 


\subsection{Weyl Operators and Gross Transform}

The Weyl operator $W(f)$ defined in (1.8) is unitary, i.e., $W^{*}(f)=W^{-1}(f)$, and satisfies the relations

$W^{-1}(f)=W(-f), \quad W(f) W(g)=W(g) W(f) e^{-2 i \operatorname{Im}\langle f, g\rangle}=W(f+g) e^{-i \operatorname{Im}\langle f, g\rangle}$

as well as the shift property

$$
W^{*}(f) a_{k} W(f)=a_{k}+f(k) .
$$

This immediately implies that the Gross transform, as defined in (2.12), is unitary. Moreover, it has the properties

$$
U_{K}=W\left(-N^{-1 / 2} \sum_{j=1}^{N} B_{K, x_{j}}\right)=\prod_{j=1}^{N} W\left(-N^{-1 / 2} B_{K, x_{j}}\right)
$$

(which holds since $\operatorname{Im}\left\langle B_{K, x}, B_{K, y}\right\rangle=0$ for all $x, y \in \mathbb{R}^{3}$ ) and

$$
U_{K} a_{k} U_{K}^{*}=a_{k}+N^{-1 / 2} \sum_{j=1}^{N} B_{K, x_{j}}(k) .
$$

\subsection{The Fröhlich Hamiltonian}

In [16], Griesemer and Wünsch give an explicit representation of $H_{N}^{\mathrm{F}}$ with the aid of the Gross transform when $N=1$. Below, we state the analogous representation for $N>1$, which will be useful for the proof of our main theorem. Considering $N>1$ does not impose additional difficulties compared to [16].

Definition 3.1. With $B_{K, x}$ and $G_{K, x}$ defined in (2.13) and (3.6), respectively, we set

$$
\begin{aligned}
A_{K, x} & =-2 i N^{-1 / 2}\left(\nabla_{x} \cdot a\left(k B_{K, x}\right)+a^{*}\left(k B_{K, x}\right) \cdot \nabla_{x}\right)+N^{-1} \Phi\left(k B_{K, x}\right)^{2}, \\
V_{K}(x-y) & =N^{-1}\left(\left\langle B_{K, x}, B_{K, y}\right\rangle+2 \operatorname{Re}\left\langle G_{x}, B_{K, y}\right\rangle\right), \\
H_{N, K}^{\mathrm{F}} & =\sum_{j=1}^{N}\left[-\Delta_{j}+N^{-1 / 2} \Phi\left(G_{K, x_{j}}\right)\right]+\mathcal{N}
\end{aligned}
$$

and define the Gross transformed Fröhlich Hamiltonian as

$$
H_{N, K}^{\mathrm{G}}=H_{N, K}^{\mathrm{F}}+\sum_{j=1}^{N} A_{K, x_{j}}+\sum_{j, l=1}^{N} V_{K}\left(x_{j}-x_{l}\right) .
$$


Note that (3.7) immediately implies the bound

$$
\left|V_{K}\left(x_{j}-x_{l}\right)\right| \leqq C K^{-1} N^{-1}
$$

for suitable $C>0$. The next result, which is the generalization of [16, Theorem 3.7] to $N \geqq 2$, justifies denoting $H_{N, K}^{\mathrm{G}}$ as Gross transformed Fröhlich Hamiltonian.

Lemma 3.1. The operator $H_{N, K}^{\mathrm{G}}$ is self-adjoint on $\mathcal{D}\left(H_{N}^{0}\right)$ for all $K>0$. Moreover, there exists a $\widetilde{K} \geqq 0$ such that for all $K \geqq \widetilde{K}$ and $N \in \mathbb{N}$, the self-adjoint operator $H_{N}^{F}$ associated to the quadratic form defined by (1.3) has the representation

$$
H_{N}^{\mathrm{F}}=U_{K}^{*} H_{N, K}^{\mathrm{G}} U_{K}, \quad \mathcal{D}\left(H_{N}^{\mathrm{F}}\right)=U_{K}^{*} \mathcal{D}\left(H_{N}^{0}\right) .
$$

We shall comment on the proof of this lemma in "Appendix A".

For use below, we also note that there is $\widetilde{K}, C>0$, such that for all $K \geqq \widetilde{K}$ and $N \geqq 1$,

$$
\begin{aligned}
& \frac{1}{2} H_{N}^{0}-C N \leqq H_{N}^{\mathrm{F}} \leqq \frac{3}{2} H_{N}^{0}+C N, \\
& \frac{1}{2} H_{N}^{0}-C N \leqq H_{N, K}^{\mathrm{G}} \leqq \frac{3}{2} H_{N}^{0}+C N
\end{aligned}
$$

hold as inequalities on the Hilbert space $L^{2}\left(\mathbb{R}^{3 N}\right) \otimes \mathcal{F}_{S}$ without symmetry constraints on the particles. This will be useful later in order to estimate expectation values with respect to wave functions that are not permutation symmetric in all particle coordinates, as e.g. in (5.8). The derivation of (3.19) and (3.20) is postponed to "Appendix A".

\section{Proof of the Main Theorem}

We first state three preliminary lemmas from which the proof of Theorem 2.1 then follows easily. The proofs of the lemmas are postponed to later sections.

If we take the limit $K \rightarrow \infty$, the Gross transform has only negligible effect on the one-particle reduced density and the coherent structure of the phonon field. This is quantified in the following lemma, whose proof is given in Sec. 7.1:

Lemma 4.1. Assume $K \geqq \widetilde{K}>0$ such that Lemma 3.1 holds. Let $\varphi \in L^{2}\left(\mathbb{R}^{3}\right)$, $\Psi_{N} \in \mathcal{D}\left(\left(H_{N}^{\mathrm{F}}\right)^{1 / 2}\right)$ with $\left\|\Psi_{N}\right\|=1$, and the Gross transform $U_{K}$ defined as in (2.12). Then,

$$
\operatorname{Tr}_{L^{2}\left(\mathbb{R}^{3}\right)}\left|\gamma_{\Psi_{N}}^{(1,0)}-\gamma_{U_{K} \Psi_{N}}^{(1,0)}\right| \leqq \frac{C}{K^{3 / 2}}\left\|\left(\frac{H_{N}^{\mathrm{F}}+C N}{N}\right)^{1 / 2} \Psi_{N}\right\|
$$

and

$$
\begin{gathered}
N^{-1}\left|\left\langle W^{*}(\sqrt{N} \varphi) \Psi_{N},\left(\mathcal{N}-U_{K}^{*} \mathcal{N} U_{K}\right) W^{*}(\sqrt{N} \varphi) \Psi_{N}\right\rangle\right| \\
\leq \frac{C\left(1+\|\varphi\|_{2}\right)}{K^{3 / 2}}\left\|\left(\frac{H_{N}^{\mathrm{F}}+C N}{N}\right)^{1 / 2} \Psi_{N}\right\|
\end{gathered}
$$

for some $C>0$. 
Next, we define a functional to compare $U_{K} \Psi_{N, t}$ with the Pekar state $\psi_{t}^{\otimes N} \otimes$ $W\left(\sqrt{N} \varphi_{t}\right) \Omega$. To this end, we introduce for $j \in\{1, \ldots, N\}$ the projections $p_{j}^{\psi}$ : $L^{2}\left(\mathbb{R}^{3 N}\right) \rightarrow L^{2}\left(\mathbb{R}^{3 N}\right)$ and $q_{j}^{\psi}: L^{2}\left(\mathbb{R}^{3 N}\right) \rightarrow L^{2}\left(\mathbb{R}^{3 N}\right)$, given by

$$
p_{j}^{\psi} f_{N}\left(x_{1}, \ldots, x_{N}\right)=\psi\left(x_{j}\right) \int d^{3} x_{j}^{\prime} \overline{\psi\left(x_{j}^{\prime}\right)} f_{N}\left(x_{1}, \ldots x_{j-1}, x_{j}^{\prime}, x_{j+1}, \ldots, x_{N}\right)
$$

for $f_{N} \in L^{2}\left(\mathbb{R}^{3 N}\right)$, and $q_{j}^{\psi}=1-p_{j}^{\psi}$. (More compactly, in bracket notation, $p_{j}^{\psi}=|\psi\rangle\left\langle\left.\psi\right|_{j}\right)$.

Definition 4.1. Let $K>0$ and $(\psi, \varphi) \in L^{2}\left(\mathbb{R}^{3}\right) \times L^{2}\left(\mathbb{R}^{3}\right)$ with $\|\psi\|=1$ and $\Psi_{N} \in \mathcal{D}\left(H_{N}^{\mathrm{F}}\right),\left\|\Psi_{N}\right\|=1$. We define $\beta_{K}^{a}: \mathcal{D}\left(H_{N}^{\mathrm{F}}\right) \times L^{2}\left(\mathbb{R}^{3}\right) \rightarrow \mathbb{R}_{0}^{+}, \beta_{K}^{b}:$ $\mathcal{D}\left(H_{N}^{\mathrm{F}}\right) \times L^{2}\left(\mathbb{R}^{3}\right) \rightarrow \mathbb{R}_{0}^{+}$and $\beta^{c}: \mathcal{D}\left(H_{N}^{\mathrm{F}}\right) \rightarrow \mathbb{R}_{0}^{+}$by

$$
\begin{aligned}
\beta_{K}^{a}\left(\Psi_{N}, \psi\right) & =\left\langle\Psi_{N}, U_{K}^{*}\left(q_{1}^{\psi} \otimes \mathbb{1}_{\mathcal{F}_{s}}\right) U_{K} \Psi_{N}\right\rangle, \\
\beta_{K}^{b}\left(\Psi_{N}, \varphi\right) & =N^{-1}\left\langle W^{*}(\sqrt{N} \varphi) U_{K} \Psi_{N}, \mathcal{N} W^{*}(\sqrt{N} \varphi) U_{K} \Psi_{N}\right\rangle \\
\beta^{c}\left(\Psi_{N}\right) & =\left\|N^{-1}\left(H_{N}^{\mathrm{F}}-\left\langle\Psi_{N}, H_{N}^{\mathrm{F}} \Psi_{N}\right\rangle\right) \Psi_{N}\right\|^{2} .
\end{aligned}
$$

Moreover, we define $\beta_{K}: \mathcal{D}\left(H_{N}^{\mathrm{F}}\right) \times L^{2}\left(\mathbb{R}^{3}\right) \times L^{2}\left(\mathbb{R}^{3}\right) \rightarrow \mathbb{R}_{0}^{+}$by

$$
\beta_{K}\left(\Psi_{N}, \psi, \varphi\right)=\beta_{K}^{a}\left(\Psi_{N}, \psi\right)+\beta_{K}^{b}\left(\Psi_{N}, \varphi\right)+\beta^{c}\left(\Psi_{N}\right) .
$$

For solutions $\Psi_{N, t}$ and $\left(\psi_{t}, \varphi_{t}\right)$ of the Schrödinger equation (1.2) and the LandauPekar equations (1.6), respectively, we use the shorthand notations

$$
\begin{aligned}
& \beta_{K}(t)=\beta_{K}\left(\Psi_{N, t}, \psi_{t}, \varphi_{t}\right), \quad \beta_{K}^{a}(t)=\beta_{K}^{a}\left(\Psi_{N, t}, \psi_{t}\right), \\
& \beta_{K}^{b}(t)=\beta_{K}^{b}\left(\Psi_{N, t}, \varphi_{t}\right), \quad \beta^{c}(t)=\beta^{c}\left(\Psi_{N, t}\right) .
\end{aligned}
$$

Remark 4.1. Note that

$$
\beta_{K}^{b}\left(\Psi_{N}, \varphi\right)=\int d^{3} k\left\|\left(N^{-1 / 2} a_{k}-\varphi(k)\right) U_{K} \Psi_{N}\right\|^{2} .
$$

Remark 4.2. $\beta_{K}(t)$ being small compared to one ensures that

- the $N$-particle component of $U_{K} \Psi_{N, t}$ is approximately given by the product $\psi_{t}^{\otimes N}$-more precisely, $\beta_{K}^{a}(t)$ measures the relative number of particles not in $\psi_{t}$,

- the phonon component of $U_{K} \Psi_{N, t}$ is close to the coherent state $W\left(\sqrt{N} \varphi_{t}\right) \Omega$ more, precisely, $\beta_{K}^{b}(t)$ measures the relative number of excitations with respect to the coherent state $W\left(\sqrt{N} \varphi_{t}\right) \Omega$,

- the variance of $N^{-1} H_{N}^{\mathrm{F}}$ with respect to $\Psi_{N, t}$ is small compared to one-this will be used to control the singular ultraviolet behavior of the phonon field (for a detailed explanation of this point, see the beginning of Section 5). Also note that $\beta^{c}\left(\Psi_{N, t}\right)=\beta^{c}\left(\Psi_{N}\right)$ is a conserved quantity, and thus requiring $\beta^{c}$ to be small only poses a restriction on the initial state. Since $\beta^{c}\left(\Psi_{N}\right)=c\left(\Psi_{N}\right)$, Proposition 2.2 shows that $\beta^{c}$ is small for initial states of the form $\Psi_{N}=$ $U_{K}^{*} \psi^{\otimes N} \otimes W(\sqrt{N} \varphi) \Omega$ with $K=K_{N}$ large enough. 
The functional $\beta_{K}(t)$ can consequently be used to monitor whether the condensate of the particles and the coherent state of the phonons is stable during the time evolution. Its definition is motivated by a previous work on the derivation of the Maxwell-Schrödinger equations [23]. In addition it is necessary to include the Gross transform in the definition of $\beta_{K}^{a}(t)$ and $\beta_{K}^{b}(t)$. This induces correlations between the electron and the phonons and effectively regularizes the interaction. In this sense, the Gross transform has a similar role as the Bogoliubov transformation in the derivation of the time-dependent Gross-Pitaevskii equation (see for instance $[2,3,20,30])$.

The trace norm of the difference $\gamma_{U_{K} \Psi_{N, t}}^{(1,0)}-\left|\psi_{t}\right\rangle\left\langle\psi_{t}\right|$ and the quantity $\beta_{K}^{a}(t)$ are related by

$$
\left.\beta_{K}^{a}(t) \leqq \operatorname{Tr}_{L^{2}\left(\mathbb{R}^{3}\right)}\left|\gamma_{U_{K} \Psi_{N, t}}^{(1,0)}-\right| \psi_{t}\right\rangle\left\langle\psi_{t}|| \leqq 4 \sqrt{\beta_{K}^{a}(t)}\right.
$$

which is the content of the following lemma when $\Psi_{N}=U_{K} \Psi_{N, t}$ :

Lemma 4.2. Let $\psi \in L^{2}\left(\mathbb{R}^{3}\right)$ with $\|\psi\|=1$ and $\Psi_{N} \in \mathcal{H}^{(N)}$ with $\left\|\Psi_{N}\right\|=1$. Then,

$$
\left.\left\langle\Psi_{N},\left(q_{1}^{\psi} \otimes \mathbb{1}_{\mathcal{F}_{s}}\right) \Psi_{N}\right\rangle \leqq \operatorname{Tr}_{L^{2}\left(\mathbb{R}^{3}\right)}\left|\gamma_{\Psi_{N}}^{(1,0)}-\right| \psi\right\rangle\left\langle\psi|| \leqq 4 \sqrt{\left\langle\Psi_{N},\left(q_{1}^{\psi} \otimes \mathbb{1}_{\mathcal{F}_{s}}\right) \Psi_{N}\right\rangle}\right.
$$

Proof. The lemma is a consequence of the identity

$$
\left.\operatorname{Tr}_{L^{2}\left(\mathbb{R}^{3}\right)}\left|\gamma_{\Psi_{N}}^{(1,0)}-\right| \psi\right\rangle\left\langle\psi||=\sup _{\|A\|_{o p}=1}\left|\left\langle\Psi_{N}, A_{1} \Psi_{N}\right\rangle-\langle\psi, A \psi\rangle\right|,\right.
$$

where the supremum is taken over all bounded operators $A: L^{2}\left(\mathbb{R}^{3}\right) \rightarrow L^{2}\left(\mathbb{R}^{3}\right)$ and

$$
A_{1}=(A \otimes \underbrace{\mathbb{1}_{L^{2}\left(\mathbb{R}^{3}\right)} \otimes \ldots \otimes \mathbb{1}_{L^{2}\left(\mathbb{R}^{3}\right)}}_{N-1 \text { times }}) \otimes \mathbb{1}_{\mathcal{F}_{s}}
$$

acts non-trivially only on the variable $x_{1}$. (Note that (4.11) holds because the space of bounded operators is the dual of the space of trace-class operators). The first bound then follows from

$$
\left\langle\Psi_{N},\left(q_{1}^{\psi} \otimes \mathbb{1}_{\mathcal{F}_{s}}\right) \Psi_{N}\right\rangle=\left\langle\Psi_{N}, q_{1}^{\psi} \Psi_{N}\right\rangle=\left|\left\langle\Psi_{N}, p_{1}^{\psi} \Psi_{N}\right\rangle-\left\langle\psi, p^{\psi} \psi\right\rangle\right|,
$$

while for the second bound, one inserts $1=p_{1}^{\psi}+q_{1}^{\psi}$ on the left and right of $A_{1}$ and uses

$$
p_{1}^{\psi} A_{1} p_{1}^{\psi}-\langle\psi, A \psi\rangle=q_{1}^{\psi}\langle\psi, A \psi\rangle
$$

together with the Cauchy-Schwarz inequality for the remaining terms. ${ }^{3}$

\footnotetext{
${ }^{3}$ From now on we omit the product with the identity and write $q_{i}^{\psi}$ and $p_{i}^{\psi}$ instead of $q_{i}^{\psi} \otimes \nVdash_{\mathcal{F}_{s}}$ and $p_{i}^{\psi} \otimes \nVdash_{\mathcal{F}_{s}}$.
} 
The main ingredient of the proof of Theorem 2.1 is the following estimate for $\beta_{K}(t)$ :

Lemma 4.3. Assume $K \geqq \widetilde{K}>0$ such that Lemma 3.1 holds. Let $\Psi_{N, t}=$ $e^{-i H_{N}^{\mathrm{F}} t} \Psi_{N}$ with $\Psi_{N} \in \mathcal{D}\left(H_{N}^{\mathrm{F}}\right)$ such that $\left\|\Psi_{N}\right\|=1$ and $E_{0}=\sup _{N \in \mathbb{N}}$ $\left|N^{-1}\left\langle\Psi_{N}, H_{N}^{\mathrm{F}} \Psi_{N}\right\rangle\right|<\infty$. Let further $\left(\psi_{t}, \varphi_{t}\right)$ be a solution of $(1.6)$ with $(\psi, \varphi) \in$ $H^{2}\left(\mathbb{R}^{3}\right) \times L_{1}^{2}\left(\mathbb{R}^{3}\right)$ such that $\|\psi\|=1$. Then, there exists a constant $C>0$ only depending on $\|\varphi\|_{L_{1}^{2}}$, $\|\psi\|_{H^{2}}$, and $E_{0}$, such that

$$
\left|\frac{\mathrm{d}}{\mathrm{d} t} \beta_{K}(t)\right| \leqq C\left(1+t^{2}\right)\left(\beta_{K}(t)+K N^{-1}+K^{-1}\right) .
$$

The proof is given in Section 6. Putting the above statements together, we obtain the proof of Theorem 2.1 .

Proof of Theorem 2.1. We first apply Grönwall's argument to (4.15) in order to obtain

$$
\beta_{K}(t) \leqq e^{C(1+|t|)^{3}}\left(\beta_{K}(0)+K N^{-1}+K^{-1}\right) .
$$

Next, set $K=K_{N}=\widetilde{K} N^{1 / 2}$ with $\widetilde{K}>0$ as in Lemma 3.1, and compute

$$
\begin{aligned}
\left.\operatorname{Tr}_{L^{2}\left(\mathbb{R}^{3}\right)}\left|\gamma_{\Psi_{N, t}}^{(1,0)}-\right| \psi_{t}\right\rangle\left\langle\psi_{t}||\right. & \left.\leqq \operatorname{Tr}_{L^{2}\left(\mathbb{R}^{3}\right)}\left|\gamma_{U_{K_{N}} \Psi_{N, t}}^{(1,0)}-\right| \psi_{t}\right\rangle\left\langle\psi_{t}||+C N^{-3 / 4}\right. \\
& \leqq 4 \sqrt{\beta_{K_{N}}^{a}(t)}+C N^{-3 / 4} \\
& \leqq 4 \sqrt{\beta_{K_{N}}(t)}+C N^{-3 / 4} \\
& \leqq \sqrt{\beta_{K_{N}}(0)+N^{-1 / 2}} e^{C(1+|t|)^{3}}
\end{aligned}
$$

where we used inequality (4.1) in the first step, Lemma (4.2) in the second and (4.16) in the last one. The estimate (2.7) then follows from $\beta^{c}(0)=c\left(\Psi_{N}\right)$ and

$$
\beta_{K_{N}}^{a}(0)+\beta_{K_{N}}^{b}(0) \leqq a\left(\Psi_{N}, \psi\right)+b\left(\Psi_{N}, \varphi\right)+C N^{-3 / 4},
$$

which in turn holds because of (4.10) and Lemma 4.1.

Using (4.2), we can similarly estimate

$$
\begin{aligned}
& N^{-1}\left\langle W^{*}\left(\sqrt{N} \varphi_{t}\right) \Psi_{N, t}, \mathcal{N} W^{*}\left(\sqrt{N} \varphi_{t}\right) \Psi_{N, t}\right\rangle \\
& \quad \leqq N^{-1}\left\langle W^{*}\left(\sqrt{N} \varphi_{t}\right) U_{K_{N}} \Psi_{N, t}, \mathcal{N} W^{*}\left(\sqrt{N} \varphi_{t}\right) U_{K_{N}} \Psi_{N, t}\right\rangle+C\left(1+\left\|\varphi_{t}\right\|\right) N^{-3 / 4} \\
& \quad=\beta_{K_{N}}^{b}(t)+C\left(1+\left\|\varphi_{t}\right\|\right) N^{-3 / 4} \\
& \quad \leqq\left(\beta_{K_{N}}(0)+N^{-1 / 2}\right) e^{C(1+|t|)^{3}} \\
& \quad \leqq\left(a\left(\Psi_{N}, \psi\right)+b\left(\Psi_{N}, \varphi\right)+c\left(\Psi_{N}\right)+N^{-1 / 2}\right) e^{C(1+|t|)^{3}} .
\end{aligned}
$$

This completes the proof of the theorem.

The proofs of Proposition 2.2 and Theorem 2.2 are postponed to Sections 7.2 and 7.3, respectively. 


\section{Bound on $\left\|\nabla_{2} q_{1}^{\psi} U_{K} \Psi_{N}\right\|$}

In this section, we state and prove a bound that is a crucial ingredient in the proof of Lemma 4.3 .

Lemma 5.1. Assume $K \geqq \widetilde{K}>0$ such that Lemma 3.1 holds. Let $(\psi, \varphi) \in$ $H^{2}\left(\mathbb{R}^{3}\right) \times L_{1}^{2}\left(\mathbb{R}^{3}\right)$ and $\Psi_{N} \in \mathcal{D}\left(H_{N}^{\mathrm{F}}\right)$ with $\left\|\Psi_{N}\right\|=1$, and set $E_{N}^{\mathrm{F}}\left(\Psi_{N}\right)=$ $N^{-1}\left\langle\Psi_{N}, H_{N}^{\mathrm{F}} \Psi_{N}\right\rangle$. Then

$$
\left\|\nabla_{2} q_{1}^{\psi} U_{K} \Psi_{N}\right\|^{2} \leqq g\left(\Psi_{N}, \psi, \varphi\right)\left(\beta_{K}\left(\Psi_{N}, \psi, \varphi\right)+N^{-1} K^{-1}+N^{-2} K\right),
$$

where $g\left(\Psi_{N}, \psi, \varphi\right)=C\left(\|\psi\|_{H^{2}}^{2}+\|\varphi\|_{L_{1}^{2}}^{2}+\left|E_{N}^{\mathrm{F}}\left(\Psi_{N}\right)\right|\right)$ for some $C>0$.

Before we give its proof, we explain the importance of the above estimate. The main technical difficulty for controlling the time-derivative of $\beta_{K}(t)$ arises from the singular ultraviolet behavior of the phonon field. In particular, if we want to estimate $\frac{\mathrm{d}}{\mathrm{d} t} \beta_{K}^{b}(t)$, we have to bound the following term (cf. Section 6.2)

$(6.21 d)=-2 \operatorname{Im}\left\langle U_{K} \Psi_{N, t}, \int_{|k| \leqq K} d^{3} k|k|^{-1} e^{i k x_{1}} q_{1}^{\psi_{t}}\left(N^{-1 / 2} a_{k}-\varphi_{t}(k)\right) U_{K} \Psi_{N, t}\right\rangle$

by an $N$-independent constant times the functional $\beta_{K}(t)$. A naive estimate using the Cauchy-Schwarz inequality would give the bound

$$
|(6.21 d)| \leqq C K^{1 / 2} \sqrt{\beta_{K}^{a}(t) \beta_{K}^{b}(t)},
$$

which is not sufficient for $K \gg 1$. The reason for the bad behavior for large $K$ clearly comes from the careless estimate of the form factor $|k|^{-1}$.

The most obvious strategy for a better estimate is to apply the well-known commutator method of Lieb and Yamazaki [26], which utilizes the particle momentum in order to obtain a better ultraviolet behavior of the phonon field. More precisely, one writes the exponential $e^{i k x_{1}}$ in terms of a commutator with the gradient $i \nabla_{x_{1}}$,

$$
e^{i k x_{1}}=\left(1+|k|^{2}\right)^{-1}\left(e^{i k x_{1}}-k \cdot\left[i \nabla_{x_{1}}, e^{i k x_{1}}\right]\right),
$$

which suggests a better decay for large $|k|$ provided that one has some control of the regularity of the particle with coordinate $x_{1}$. Using this identity together with $p_{1}^{\psi_{t}}+q_{1}^{\psi_{t}}=1$, we find by a straightforward computation that (6.21d) can be written as

$$
\begin{aligned}
&-2 \operatorname{Im} \int_{|k| \leqq K} d^{3} k|k|^{-1}\left(1+|k|^{2}\right)^{-1} \\
& \times\left(\left\langle e^{-i k x_{1}}\left(N^{-1 / 2} a_{k}^{*}-\overline{\varphi_{t}(k)}\right) U_{K} \Psi_{N, t}, q_{1}^{\psi_{t}} U_{K} \Psi_{N, t}\right\rangle\right. \\
&-\left\langle e^{-i k x_{1}}\left(N^{-1 / 2} a_{k}^{*}-\overline{\varphi_{t}(k)}\right) k \cdot i \nabla_{1} p_{1}^{\psi_{t}} U_{K} \Psi_{N, t}, q_{1}^{\psi_{t}} U_{K} \Psi_{N, t}\right\rangle
\end{aligned}
$$




$$
\begin{aligned}
& -\left\langle e^{-i k x_{1}} k \cdot i \nabla_{1} q_{1}^{\psi_{t}} U_{K} \Psi_{N, t},\left(N^{-1 / 2} a_{k}-\varphi_{t}(k)\right) q_{1}^{\psi_{t}} U_{K} \Psi_{N, t}\right\rangle \\
& \left.+\left\langle e^{-i k x_{1}}\left(N^{-1 / 2} a_{k}^{*}-\overline{\varphi_{t}(k)}\right) U_{K} \Psi_{N, t}, k \cdot i \nabla_{1} q_{1}^{\psi_{t}} U_{K} \Psi_{N, t}\right\rangle\right) .
\end{aligned}
$$

With the aid of the Cauchy-Schwarz inequality and the canonical commutation relations this implies the bound

$$
|(6.21 d)| \leqq C\left(\left\|\psi_{t}\right\|_{H^{1}} \sqrt{\beta_{K}^{a}(t)}+\left\|\nabla_{1} q_{1}^{\psi_{t}} U_{K} \Psi_{N, t}\right\|\right) \sqrt{\beta_{K}^{b}(t)+N^{-1}} .
$$

Contrary to (5.3), there is no more divergence for large $K$. However, the above inequality contains the new term $\left\|\nabla_{1} q_{1}^{\psi_{t}} U_{K} \Psi_{N, t}\right\|$. Thus if we want to apply Grönwall's inequality we would have to show that this term is small compared to one or bounded by a constant times $\sqrt{\beta_{K}(t)} .{ }^{4}$ It is not clear how to derive such a bound, however, and hence, we are forced to estimate $|(6.21 d)|$ in a different way.

A possible solution to this problem is to use a combination of the estimates from [23, Chapter VIII.1] with an operator bound that is motivated by [10, Lemma 10] (see Section 6 for the detailed argument). In short, we use the symmetry of the wave function and an estimate that is similar in spirit to the commutator method of Lieb and Yamazaki to obtain

$$
|(6.21 d)| \leqq C\left(\beta_{K}^{a}(t)+\beta_{K}^{b}(t)+N^{-1} K+\left\|\nabla_{2} q_{1}^{\psi_{t}} U_{K} \Psi_{N, t}\right\|^{2}\right) .
$$

As shown in Lemma 5.1, the new quantity $\left\|\nabla_{2} q_{1}^{\psi_{t}} U_{K} \Psi_{N, t}\right\|^{2}$ can be bounded by $\beta_{K}(t)$ and errors proportional to $N^{-1} K^{-1}$ and $N^{-2} K$.

Proof of Lemma 5.1. Using the symmetry of $\Psi_{N}$ and $-\Delta_{1} \geqq 0$, we can bound

$$
\begin{aligned}
\left\|\nabla_{2} q_{1}^{\psi} U_{K} \Psi_{N}\right\|^{2} & =(N-1)^{-1} \sum_{j=2}^{N}\left\langle q_{1}^{\psi} U_{K} \Psi_{N},\left(-\Delta_{j}\right) q_{1}^{\psi} U_{K} \Psi_{N}\right\rangle \\
& \leqq \frac{2}{N} \sum_{j=1}^{N}\left\langle q_{1}^{\psi} U_{K} \Psi_{N},\left(-\Delta_{j}\right) q_{1}^{\psi} U_{K} \Psi_{N}\right\rangle .
\end{aligned}
$$

With $-\sum_{j=1}^{N} \Delta_{j} \leqq H_{N}^{0}$ and (3.20), we thus have

$$
\left\|\nabla_{2} q_{1}^{\psi} U_{K} \Psi_{N}\right\|^{2} \leqq C \beta_{K}^{a}\left(\Psi_{N}, \psi\right)+4 N^{-1}\left\langle q_{1}^{\psi} U_{K} \Psi_{N}, H_{N, K}^{\mathrm{G}} q_{1}^{\psi} U_{K} \Psi_{N}\right\rangle .
$$

By using $q_{1}^{\psi} H_{N, K}^{\mathrm{G}} q_{1}^{\psi}=q_{1}^{\psi} H_{N, K}^{\mathrm{G}}-q_{1}^{\psi} H_{N, K}^{\mathrm{G}} p_{1}^{\psi}$ and recalling Definition (3.1), we get

$$
\left\|\nabla_{2} q_{1}^{\psi} U_{K} \Psi_{N}\right\|^{2} \leqq C\left(\beta_{K}^{a}\left(\Psi_{N}, \psi\right)+N^{-1}\left|\left\langle q_{1}^{\psi} U_{K} \Psi_{N}, H_{N, K}^{\mathrm{G}} U_{K} \Psi_{N}\right\rangle\right|\right.
$$

\footnotetext{
${ }^{4}$ We note that the quantity $\left\|\nabla_{1} q_{1}^{\psi_{t}} U_{K} \Psi_{N, t}\right\|^{2}$ can be related to the Sobolev trace norm difference between the one-particle reduced density matrix and the condensate wave function (see [27, Proof of Theorem 2.8] and [24, Lemma 7.1]).
} 


$$
\begin{aligned}
& +N^{-1}\left|\left\langle U_{K} \Psi_{N}, q_{1}^{\psi}\left(-\Delta_{1}\right) p_{1}^{\psi} U_{K} \Psi_{N}\right\rangle\right| \\
& +N^{-3 / 2}\left|\left\langle U_{K} \Psi_{N}, q_{1}^{\psi} \Phi\left(G_{K, x_{1}}\right) p_{1}^{\psi} U_{K} \Psi_{N}\right\rangle\right| \\
& +N^{-1}\left|\left\langle U_{K} \Psi_{N}, q_{1}^{\psi} A_{K, x_{1}} p_{1}^{\psi} U_{K} \Psi_{N}\right\rangle\right| \\
& \left.+\left|\left\langle U_{K} \Psi_{N}, q_{1}^{\psi} V_{K}\left(x_{1}-x_{2}\right) p_{1}^{\psi} U_{K} \Psi_{N}\right\rangle\right|\right) .
\end{aligned}
$$

In what follows, we shall bound the various terms on the right hand side.

Line (5.10a). In the second summand in this line, we add and subtract $E_{N}^{\mathrm{F}}\left(\Psi_{N}\right)$ $\beta_{K}^{a}\left(\Psi_{N}, \varphi\right)$, to obtain

$$
\begin{aligned}
& N^{-1}\left|\left\langle q_{1}^{\psi} U_{K} \Psi_{N}, H_{N, K}^{\mathrm{G}} U_{K} \Psi_{N}\right\rangle\right| \\
& \quad \leqq\left|\left\langle q_{1}^{\psi} U_{K} \Psi_{N},\left(N^{-1} H_{N, K}^{\mathrm{G}}-E_{N}^{\mathrm{F}}\left(\Psi_{N}\right)\right) U_{K} \Psi_{N}\right\rangle\right|+E_{N}^{\mathrm{F}}\left(\Psi_{N}\right) \beta_{K}^{a}\left(\Psi_{N}, \psi\right) .
\end{aligned}
$$

With the aid of the Cauchy-Schwarz inequality and (3.18), we find

$$
|(5.10 a)| \leqq C\left(1+\left|E_{N}^{\mathrm{F}}\left(\Psi_{N}\right)\right|\right)\left(\beta_{K}^{a}\left(\Psi_{N}, \psi\right)+\beta^{c}\left(\Psi_{N}\right)\right) .
$$

Line (5.10b). One readily obtains

$$
\begin{aligned}
(5.10 b) & \leqq N^{-1}\left\|\left(-\Delta_{1}\right) p_{1}^{\psi} U_{K} \Psi_{N}\right\|\left\|q_{1}^{\psi} U_{K} \Psi_{N}\right\| \\
& \leqq \frac{1}{2}\|\psi\|_{H^{2}}\left(\beta_{K}^{a}\left(\Psi_{N}, \psi\right)+N^{-2}\right) .
\end{aligned}
$$

Line (5.10c). Using (3.8), we find

$$
\begin{aligned}
(5.10 c) & \leqq N^{-3 / 2}\left\|q_{1}^{\psi} U_{K} \Psi_{N}\right\|\left\|\Phi\left(G_{K, x_{1}}\right) p_{1}^{\psi} U_{K} \Psi_{N}\right\| \\
& \leqq C N^{-3 / 2} K^{1 / 2} \sqrt{\beta_{K}^{a}\left(\Psi_{N}, \psi\right)\left\langle U_{K} \Psi_{N},(\mathcal{N}+1) U_{K} \Psi_{N}\right\rangle}
\end{aligned}
$$

and hence, using (3.20), (3.18) and $\mathcal{N} \leqq H_{N}^{0}$, we have

$$
(5.10 c) \leqq C\left(1+\left|E_{N}^{\mathrm{F}}\left(\Psi_{N}\right)\right|\right)\left(K N^{-2}+\beta_{K}^{a}\left(\Psi_{N}, \psi\right)\right) .
$$

Line (5.10d). We recall the definition of $A_{K, x}$ in (3.13) and estimate the term with $a^{*}\left(k B_{K, x}\right) \cdot \nabla_{x}$ by

$$
\begin{aligned}
& \left|\left\langle U_{K} \Psi_{N}, q_{1}^{\psi} N^{-1 / 2} a^{*}\left(k B_{K, x_{1}}\right) \cdot \nabla_{1} p_{1}^{\psi} U_{K} \Psi_{N}\right\rangle\right| \\
& \quad \leqq \int d^{3} k\left|\left\langle q_{1}^{\psi}\left(N^{-1 / 2} a_{k}-\varphi(k)+\varphi(k)\right) U_{K} \Psi_{N}, k B_{K, x_{1}}(k) \cdot \nabla_{1} p_{1}^{\psi} U_{K} \Psi_{N}\right\rangle\right| \\
& \leqq\left\|\nabla_{1} p_{1}^{\psi} U_{K} \Psi_{N}\right\| \int d^{3} k\left(\left|k B_{K, x}(k)\right|\left\|\left(N^{-1 / 2} a_{k}-\varphi(k)\right) U_{K} \Psi_{N}\right\|+\left|k B_{K, x}(k) \varphi(k)\right|\right) \\
& \quad \leqq\|\psi\|_{H^{1}}\left(\left\||\cdot| B_{K, x}\right\| \sqrt{\beta_{K}^{b}\left(\Psi_{N}, \varphi\right)}+\left\|B_{K, x}\right\|\||\cdot| \varphi\|\right)
\end{aligned}
$$




$$
\leqq C\|\psi\|_{H^{1}}\left(K^{-1 / 2} \sqrt{\beta_{K}^{b}\left(\Psi_{N}, \varphi\right)}+K^{-3 / 2}\|\varphi\|_{L_{1}^{2}}\right)
$$

Using $q_{1}^{\psi}=1-p_{1}^{\psi}$ and $-\Delta_{1} \leqq N^{-1} H_{N}^{0}$ as quadratic forms on $L_{S}^{2}\left(\mathbb{R}^{3 N}\right) \otimes \mathcal{F}_{S}$, together with (3.20), we find

$$
\left\|\nabla_{1} q_{1}^{\psi} U_{K} \Psi_{N}\right\|^{2} \leqq 2\left(\left\|\nabla_{1} p_{1}^{\psi} U_{K} \Psi_{N}\right\|^{2}+\left\|\nabla_{1} U_{K} \Psi_{N}\right\|^{2}\right) \leqq C\left(\|\psi\|_{H^{1}}^{2}+\left|E_{N}^{\mathrm{F}}\left(\Psi_{N}\right)\right|+1\right) .
$$

With this at hand, we can proceed for the term with $\nabla_{x} \cdot a\left(k B_{K, x}\right)$ similarly as in (5.16), with the result that

$$
\begin{aligned}
& \left|\left\langle U_{K} \Psi_{N}, q_{1}^{\psi} \nabla_{1} \cdot N^{-1 / 2} a\left(k B_{K, x_{1}}\right) p_{1}^{\psi} U_{K} \Psi_{N}\right\rangle\right| \\
& \leqq\left\|\nabla_{1} q_{1}^{\psi} U_{K} \Psi_{N}\right\| \int d^{3} k\left(\left|k B_{K, x}(k)\right|\left\|\left(N^{-1 / 2} a_{k}-\varphi(k)\right) U_{K} \Psi_{N}\right\|+\left|k B_{K, x}(k) \varphi(k)\right|\right) \\
& \leqq C \sqrt{\|\psi\|_{H^{1}}^{2}+\left|E_{N}^{\mathrm{F}}\left(\Psi_{N}\right)\right|}\left(K^{-1 / 2} \sqrt{\beta_{K}^{b}\left(\Psi_{N}, \varphi\right)}+K^{-3 / 2}\|\varphi\|_{L_{1}^{2}}\right)
\end{aligned}
$$

Next, we estimate the term in line (5.10d) with $\Phi\left(k B_{K, x}\right)^{2}$,

$$
\begin{aligned}
& \left|\left\langle U_{K} \Psi_{N}, q_{1}^{\psi} N^{-1} \Phi\left(k B_{K, x_{1}}\right)^{2} p_{1}^{\psi} U_{K} \Psi_{N}\right\rangle\right| \\
& \quad \leqq N^{-1}\left\|\Phi\left(k B_{K, x_{1}}\right) q_{1}^{\psi} U_{K} \Psi_{N}\right\|\left\|\Phi\left(k B_{K, x_{1}}\right) p_{1}^{\psi} U_{K} \Psi_{N}\right\| \\
& \leq C N^{-1}\left\||\cdot| B_{K, x}\right\|^{2}\left\langle U_{K} \Psi_{N},(\mathcal{N}+1) U_{K} \Psi_{N}\right\rangle \\
& \leq C K^{-1} N^{-1}\left(\left\langle U_{K} \Psi_{N},\left(H_{N, K}^{\mathrm{G}}+C N\right) U_{K} \Psi_{N}\right\rangle+1\right) \\
& \leqq C\left(\left|E_{N}^{\mathrm{F}}\left(\Psi_{N}\right)\right|+1\right) K^{-1} .
\end{aligned}
$$

By summing up the terms, we obtain the bound

$$
\begin{aligned}
& \left|\left\langle U_{K} \Psi_{N}, q_{1}^{\psi} A_{K, x_{1}} p_{1}^{\psi} U_{K} \Psi_{N}\right\rangle\right| \\
& \quad \leqq C\left(\|\psi\|_{H^{1}}^{2}+\|\varphi\|_{L_{1}^{2}}^{2}+\left|E_{N}^{\mathrm{F}}\left(\Psi_{N}\right)\right|\right)\left(K^{-1}+\beta_{K}^{b}\left(\Psi_{N}, \varphi\right)\right) .
\end{aligned}
$$

Line (5.10e). Using (3.17),

$$
\begin{aligned}
\left|\left\langle U_{K} \Psi_{N}, q_{1}^{\psi} V_{K}\left(x_{1}-x_{2}\right) p_{1}^{\psi} U_{K} \Psi_{N}\right\rangle\right| & \leqq \sqrt{\beta_{K}^{a}\left(\Psi_{N}, \psi\right)}\left\|V_{K}\left(x_{1}-x_{2}\right) p_{1}^{\psi} U_{K} \Psi_{N}\right\| \\
& \leqq C\left(\beta_{K}^{a}\left(\Psi_{N}, \psi\right)+N^{-2} K^{-2}\right) .
\end{aligned}
$$

This completes the proof of the lemma. 


\section{Proof of Lemma 4.3 (Time Derivative of $\beta_{K}(t)$ )}

We first observe that

$$
\frac{\mathrm{d}}{\mathrm{d} t} U_{K} \Psi_{N, t}=-i U_{K} H_{N}^{\mathrm{F}} \Psi_{N, t}=-i U_{K} H_{N}^{\mathrm{F}} U_{K}^{*} U_{K} \Psi_{N, t}=-i H_{N, K}^{\mathrm{G}} U_{K} \Psi_{N, t},
$$

from which it follows readily that $\frac{\mathrm{d}}{\mathrm{d} t} \beta^{c}(t)=0$. The time-derivatives of $\beta_{K}^{a}(t)$ and $\beta_{K}^{b}(t)$ are estimated in the next two sections. Throughout both sections, we use the abbreviation $E_{N}^{\mathrm{F}}\left(\Psi_{N}\right)=N^{-1}\left\langle\Psi_{N}, H_{N}^{\mathrm{F}} \Psi_{N}\right\rangle$.

\subsection{Time Derivative of $\beta_{K}^{a}(t)$}

For $q_{1}^{t}=q_{1}^{\psi_{t}}=1-p_{1}^{\psi_{t}}$, we have

$$
\frac{\mathrm{d}}{\mathrm{d} t} q_{1}^{t}=-\frac{\mathrm{d}}{\mathrm{d} t} p_{1}^{t}=i\left[-\Delta_{1}+\Phi\left(x_{1}, t\right), p_{1}^{t}\right]=-i\left[-\Delta_{1}+\Phi\left(x_{1}, t\right), q_{1}^{t}\right] .
$$

Using this together with (6.1), we compute

$$
\begin{aligned}
\frac{\mathrm{d}}{\mathrm{d} t} \beta_{K}^{a}(t) & =\frac{\mathrm{d}}{\mathrm{d} t}\left\langle U_{K} \Psi_{N, t}, q_{1}^{t} U_{K} \Psi_{N, t}\right\rangle \\
& =-2 \operatorname{Im}\left\langle U_{K} \Psi_{N, t},\left(H_{N, K}^{\mathrm{G}}+\Delta_{1}-\Phi\left(x_{1}, t\right)\right) q_{1}^{t} U_{K} \Psi_{N, t}\right\rangle \\
& =-2 \operatorname{Im}\left\langle U_{K} \Psi_{N, t}, p_{1}^{t}\left(H_{N, K}^{\mathrm{G}}+\Delta_{1}-\Phi\left(x_{1}, t\right)\right) q_{1}^{t} U_{K} \Psi_{N, t}\right\rangle,
\end{aligned}
$$

where we inserted $1=p_{1}^{t}+q_{1}^{t}$ and used that the term with $q_{1}^{t}$ on both sides is real. Recall Definition 3.1. Using $\Phi\left(x_{1}, t\right)=\Phi_{K}\left(x_{1}, t\right)+\Phi_{\geqq K}\left(x_{1}, t\right), p_{1}^{t} q_{1}^{t}=0$ and the symmetry of $\Psi_{N}$, we can rewrite (6.3) as

$$
\begin{aligned}
\frac{\mathrm{d}}{\mathrm{d} t} \beta_{K}^{a}(t)= & -2 \operatorname{Im}\left\langle U_{K} \Psi_{N, t}, p_{1}^{t}\left(N^{-1 / 2} \Phi\left(G_{K, x_{1}}\right)-\Phi_{K}\left(x_{1}, t\right)\right) q_{1}^{t} U_{K} \Psi_{N, t}\right\rangle \\
& +2 \operatorname{Im}\left\langle U_{K} \Psi_{N, t}, p_{1}^{t} \Phi_{\geqq K}\left(x_{1}, t\right) q_{1}^{t} U_{K} \Psi_{N, t}\right\rangle \\
& -2 \operatorname{Im}\left\langle U_{K} \Psi_{N, t}, p_{1}^{t} A_{K, x_{1}} q_{1}^{t} U_{K} \Psi_{N, t}\right\rangle \\
& -2 \operatorname{Im}\left\langle U_{K} \Psi_{N, t}, p_{1}^{t}(N-1) V_{K}\left(x_{1}-x_{2}\right) q_{1}^{t} U_{K} \Psi_{N, t}\right\rangle .
\end{aligned}
$$

The various terms will be bounded as follows:

Line (6.4a). We bound

$$
\begin{aligned}
|(6.4 a)| \leqq & 2\left|\left\langle\int_{|k| \leqq K} d^{3} k|k|^{-1} e^{-i k x_{1}}\left(N^{-1 / 2} a_{k}^{*}-\overline{\varphi_{t}(k)}\right) p_{1}^{t} U_{K} \Psi_{N, t}, q_{1}^{t} U_{K} \Psi_{N, t}\right\rangle\right| \\
& +2\left|\left\langle\int_{|k| \leqq K} d^{3} k|k|^{-1} e^{i k x_{1}}\left(N^{-1 / 2} a_{k}-\varphi_{t}(k)\right) p_{1}^{t} U_{K} \Psi_{N, t}, q_{1}^{t} U_{K} \Psi_{N, t}\right\rangle\right| \\
\leqq & 2 \beta_{K}^{a}\left(\Psi_{N, t}, \psi_{t}\right)+\left\|\int_{|k| \leqq K} d^{3} k|k|^{-1} e^{-i k x_{1}}\left(N^{-1 / 2} a_{k}^{*}-\overline{\varphi_{t}(k)}\right) p_{1}^{t} U_{K} \Psi_{N, t}\right\|^{2}
\end{aligned}
$$




$$
+\left\|\int_{|k| \leqq K} d^{3} k|k|^{-1} e^{i k x_{1}}\left(N^{-1 / 2} a_{k}-\varphi_{t}(k)\right) p_{1}^{t} U_{K} \Psi_{N, t}\right\|^{2} .
$$

For the second summand, we use

$$
\begin{aligned}
& \left\|\int_{|k| \leqq K} d^{3} k|k|^{-1} e^{-i k x_{1}}\left(N^{-1 / 2} a_{k}^{*}-\overline{\varphi_{t}(k)}\right) p_{1}^{t} U_{K} \Psi_{N, t}\right\|^{2} \\
& \quad \leqq \frac{C K}{N}+\left\|\int_{|k| \leqq K} d^{3} k|k|^{-1} e^{i k x_{1}}\left(N^{-1 / 2} a_{k}-\varphi_{t}(k)\right) p_{1}^{t} U_{K} \Psi_{N, t}\right\|^{2},
\end{aligned}
$$

which follows directly from the canonical commutation relations. By the shift property (3.10), the last summand in (6.5) can be written as

$$
N^{-1}\left\|a\left(G_{K, x_{1}}\right) p_{1}^{t} W^{*}\left(\sqrt{N} \varphi_{t}\right) U_{K} \Psi_{N, t}\right\|^{2} .
$$

In order to estimate this expression, we use [10, Lemma 10] which implies the bound

$$
a^{*}\left(G_{K, x_{1}}\right) a\left(G_{K, x_{1}}\right) \leqq C_{G}\left(1-\Delta_{1}\right) \mathcal{N},
$$

with

$$
C_{G}=\sup _{p \in \mathbb{R}^{3}} \int_{\mathbb{R}^{3}} \frac{d^{3} k}{k^{2}\left(1+(p+k)^{2}\right)}=\int_{\mathbb{R}^{3}} \frac{d^{3} k}{k^{2}(1+k)^{2}}<\infty .
$$

The latter is obtained via a rearrangement inequality. In combination, we thus have

$$
\begin{aligned}
|(6.4 a)| & \leqq C\left(\beta_{K}^{a}(t)+K N^{-1}\right)+C_{G} N^{-1}\left\|\left(1-\Delta_{1}\right)^{1 / 2} p_{1}^{t} \mathcal{N}^{1 / 2} W^{*}\left(\sqrt{N} \varphi_{t}\right) U_{K} \Psi_{N, t}\right\|^{2} \\
& \leqq C\left(\beta_{K}^{a}(t)+K N^{-1}\right)+C_{G}\left\|\psi_{t}\right\|_{H^{1}}^{2} N^{-1}\left\|\mathcal{N}^{1 / 2} W^{*}\left(\sqrt{N} \varphi_{t}\right) U_{K} \Psi_{N, t}\right\|^{2} \\
& \leqq C\left\|\psi_{t}\right\|_{H^{1}}^{2}\left(\beta_{K}^{a}(t)+\beta_{K}^{b}(t)+K N^{-1}\right)
\end{aligned}
$$

Line (6.4b). This term can be estimated as

$$
\begin{aligned}
|(6.4 b)| & \leqq C \sup _{x}\left|\Phi_{\geq K}(x, t)\right|\left\|q_{1}^{t} U_{K} \Psi_{N, t}\right\| \\
& \leqq C \sqrt{\beta_{K}^{a}(t)} \int_{|k| \geqq K} d^{3} k|k|^{-1}\left|\varphi_{t}\right| \\
& \leqq C \sqrt{\beta_{K}^{a}(t)}\left\|\varphi_{t}\right\|_{L_{1}^{2}}\left(\int_{|k| \geqq K} d^{3} k|k|^{-4}\right)^{1 / 2} \leqq C\left\|\varphi_{t}\right\|_{L_{1}^{2}} \sqrt{\beta_{K}^{a}(t)} K^{-1 / 2} .
\end{aligned}
$$

Line (6.4c). It follows from (5.20) that

$$
|(6.4 c)| \leqq C\left(\left\|\psi_{t}\right\|_{H^{1}}^{2}+\left\|\varphi_{t}\right\|_{L_{1}^{2}}^{2}+\left|E_{N}^{\mathrm{F}}\left(\Psi_{N, t}\right)\right|\right)\left(K^{-1}+\beta_{K}^{b}(t)\right) .
$$


Line (6.4d). In analogy to (5.21) one finds

$$
|(6.4 d .)| \leqq C\left(\beta_{K}^{a}(t)+K^{-2}\right) .
$$

In combination, we have thus shown that

$$
\left|\frac{\mathrm{d}}{\mathrm{d} t} \beta_{K}^{a}(t)\right| \leqq C\left(\left\|\psi_{t}\right\|_{H^{1}}^{2}+\left\|\varphi_{t}\right\|_{L_{1}^{2}}^{2}+\left|E_{N}^{\mathrm{F}}\left(\Psi_{N, t}\right)\right|\right)\left(\beta_{K}^{a}(t)+\beta_{K}^{b}(t)+\frac{K}{N}+K^{-1}\right) .
$$

\subsection{Time Derivative of $\beta_{K}^{b}(t)$}

From (6.1) we get

$$
\begin{aligned}
& \frac{\mathrm{d}}{\mathrm{d} t} \beta_{K}^{b}\left(\Psi_{N, t}, \varphi_{t}\right) \\
& =\int d^{3} k \frac{\mathrm{d}}{\mathrm{d} t}\left\langle\left(N^{-1 / 2} a_{k}-\varphi_{t}(k)\right) U_{K} \Psi_{N, t},\left(N^{-1 / 2} a_{k}-\varphi_{t}(k)\right) U_{K} \Psi_{N, t}\right\rangle \\
& =-2 \operatorname{Re} \int d^{3} k\left\langle\left(N^{-1 / 2} a_{k}^{*}-\overline{\varphi_{t}(k)}\right)\left(N^{-1 / 2} a_{k}-\varphi_{t}(k)\right) U_{K} \Psi_{N, t}, i H_{N, K}^{\mathrm{G}} U_{K} \Psi_{N, t}\right\rangle \\
& \quad-2 \operatorname{Re} \int d^{3} k\left\langle\left(\partial_{t} \varphi_{t}(k)\right) U_{K} \Psi_{N, t},\left(N^{-1 / 2} a_{k}-\varphi_{t}(k)\right) U_{K} \Psi_{N, t}\right\rangle,
\end{aligned}
$$

which is a slightly formal computation, since the use of the product rule of differentiation is not completely obvious here. We clarify the difficulty and justify the above identity in detail in "Appendix B". Next, we write the first line in terms of the commutator as

$$
=-i \int d^{3} k\left\langle\left[H_{N, K}^{\mathrm{G}},\left(N^{-1 / 2} a_{k}^{*}-\overline{\varphi_{t}(k)}\right)\left(N^{-1 / 2} a_{k}-\varphi_{t}(k)\right)\right] U_{K} \Psi_{N, t}, U_{K} \Psi_{N, t}\right\rangle .
$$

Let us remark that the right hand side is well defined since the commutator of $H_{N, K}^{\mathrm{G}}$ and

$$
L_{N}=N^{-1} W\left(\sqrt{N} \varphi_{t}\right) \mathcal{N} W^{*}\left(\sqrt{N} \varphi_{t}\right)=\int d^{3} k\left(N^{-1 / 2} a_{k}^{*}-\overline{\varphi_{t}(k)}\right)\left(N^{-1 / 2} a_{k}-\varphi_{t}(k)\right)
$$

defines a bounded operator from $\mathcal{D}\left(H_{N, K}^{\mathrm{G}}\right)=\mathcal{D}\left(H_{N}^{0}\right)$ to $\mathcal{H}^{(N)}$. This fact is a direct consequence of the B.L.T. theorem because $\left[H_{N, K}^{\mathrm{G}}, L_{N}\right]$ is a bounded operator from $\mathcal{D}\left(\left(H_{N}^{0}\right)^{2}\right)$ to $\mathcal{H}^{(N)}$ and the estimate

$$
\left\|\left[H_{N, K}^{\mathrm{G}}, L_{N}\right] \Psi_{N}\right\| \leqq C_{N, K}\left\|\left(1+H_{N}^{0}\right) \Psi_{N}\right\|
$$


holds for all $\Psi_{N} \in \mathcal{D}\left(\left(H_{N}^{0}\right)^{2}\right)$ and some constant $C_{N, K}$. The latter is straightforward to verify with the aid of

$$
\begin{aligned}
{\left[H_{N, K}^{\mathrm{G}}, a_{k}\right]=} & 2 N^{-1 / 2} \sum_{j=1}^{N} B_{K, x_{j}}(k) k \cdot\left(i \nabla_{j}-N^{-1 / 2} \Phi\left(k B_{K, x_{j}}\right)\right) \\
& -a_{k}-N^{-1 / 2} \sum_{j=1}^{N}|k|^{-1} \mathbb{1}_{|k| \leqq K}(k) e^{-i k x_{j}}
\end{aligned}
$$

and the basic estimates from Section 3.

Hence, we can proceed by using (6.16) and (6.19) together with the LandauPekar equations (1.6) and the symmetry of the many-body wave function in order to obtain

$$
\begin{aligned}
\frac{\mathrm{d}}{\mathrm{d} t} \beta_{K}^{b}\left(\Psi_{N, t}, \varphi_{t}\right) \\
=-2 \int_{|k| \leqq K} d^{3} k|k|^{-1} \operatorname{Im}\left\langle e^{-i k x_{1}} U_{K} \Psi_{N, t},\left(N^{-1 / 2} a_{k}-\varphi_{t}(k)\right) U_{K} \Psi_{N, t}\right\rangle \\
\quad+2 \int d^{3} k|k|^{-1} \operatorname{Im}\left\langle\int d^{3} y e^{-i k y}\left|\psi_{t}(y)\right|^{2} U_{K} \Psi_{N, t},\left(N^{-1 / 2} a_{k}-\varphi_{t}(k)\right) U_{K} \Psi_{N, t}\right\rangle \\
\quad+4 \int d^{3} k \operatorname{Im}\left\langle B_{K, x_{1}}(k) k \cdot\left(i \nabla_{1}-N^{-1 / 2} \Phi\left(k B_{K, x_{1}}\right)\right) U_{K} \Psi_{N, t},\left(N^{-1 / 2} a_{k}-\varphi_{t}(k)\right) U_{K} \Psi_{N, t}\right\rangle .
\end{aligned}
$$

Finally, inserting the identity $e^{i k x_{1}}=p_{1}^{t} e^{i k x_{1}} p_{1}^{t}+q_{1}^{t} e^{i k x_{1}} p_{1}^{t}+e^{i k x_{1}} q_{1}^{t}$ leads to

$$
\begin{aligned}
& \frac{\mathrm{d}}{\mathrm{d} t} \beta_{K}^{b}\left(\Psi_{N, t}, \varphi_{t}\right) \\
& =-2 \int_{|k| \leqq K} d^{3} k|k|^{-1} \operatorname{Im}\left\langle U_{K} \Psi_{N, t},\left(p_{1}^{t} e^{i k x_{1}} p_{1}^{t}-\int d^{3} y e^{i k y}\left|\psi_{t}(y)\right|^{2}\right)\right. \\
& \quad+2 \int_{|k| \geqq K} d^{3} k|k|^{-1} \operatorname{Im}\left\langle U_{K} \Psi_{N, t}, \int d^{3} y e^{i k y}\left|\psi_{t}(y)\right|^{2}\left(N^{-1 / 2} a_{k}-\varphi_{t}(k)\right) U_{K} \Psi_{N, t}\right\rangle \\
& \quad-2 \int_{|k| \leqq K} d^{3} k|k|^{-1} \operatorname{Im}\left\langle U_{K} \Psi_{N, t}, q_{1}^{t} e^{i k x_{1}} p_{1}^{t}\left(N^{-1 / 2} a_{k}-\varphi_{t}(k)\right) U_{K} \Psi_{N, t}\right\rangle \\
& \quad-2 \int_{|k| \leqq K} d^{3} k|k|^{-1} \operatorname{Im}\left\langle U_{K} \Psi_{N, t}, e^{i k x_{1}} q_{1}^{t}\left(N^{-1 / 2} a_{k}-\varphi_{t}(k)\right) U_{K} \Psi_{N, t}\right\rangle \\
& \left.\quad+4 \int_{d^{3} k \operatorname{Im}\left\langle B_{K, x_{1}}(k) k \cdot\left(i \nabla_{1}-N^{-1 / 2} \Phi\left(k B_{K, x_{1}}\right)\right)\right.} U_{K} \Psi_{N, t},\left(N^{-1 / 2} a_{k}-\varphi_{t}(k)\right) U_{K} \Psi_{N, t}\right\rangle
\end{aligned}
$$

In what follows, we estimate each term on the r.h.s. separately. 
Line (6.21a). The first term is the most important because it is the one where the particle density cancels the source term of the Landau-Pekar equations. We first note that

$$
p_{1}^{t} e^{i k x_{1}} p_{1}^{t}-\int d^{3} y e^{i k y}\left|\psi_{t}(y)\right|^{2}=\left(p_{1}^{t}-1\right) \int d^{3} y e^{i k y}\left|\psi_{t}(y)\right|^{2}=-q_{1}^{t}\left|\psi_{t}, e^{i k \cdot} \psi_{t}\right\rangle .
$$

We then use $e^{i k x}=\frac{1-i\left(k \cdot \nabla_{x}\right)}{1+k^{2}} e^{i k x}$ and integrate by parts to obtain the bound

$$
\begin{aligned}
\left|\left\langle\psi_{t}, e^{i k \cdot} \psi_{t}\right\rangle\right| & \leqq\left|\left\langle\frac{1-i(k \cdot \nabla)}{1+k^{2}} \psi_{t}, e^{i k \cdot} \psi_{t}\right\rangle\right|+\left|\left\langle\psi_{t}, e^{i k \cdot} \frac{i(k \cdot \nabla)}{1+k^{2}} \psi_{t}\right\rangle\right| \\
& \leqq 2\left(1+k^{2}\right)^{-1}\left(1+|k|\left\|\nabla \psi_{t}\right\|\right) \leqq 2\left\|\psi_{t}\right\|_{H^{1}}\left(1+k^{2}\right)^{-1}(1+|k|) .
\end{aligned}
$$

Hence,

$$
\begin{aligned}
|(6.21 a)| \leqq & 4\left\|\psi_{t}\right\|_{H^{1}} \int d^{3} k \frac{(1+|k|)}{|k|\left(1+k^{2}\right)}\left|\left\langle q_{1}^{t} U_{K} \Psi_{N, t},\left(N^{-1 / 2} a_{k}-\varphi_{t}(k)\right) U_{K} \Psi_{N, t}\right\rangle\right| \\
\leqq & 4\left\|\psi_{t}\right\|_{H^{1}} \sqrt{\beta_{K}^{a}(t)}\left(\int d^{3} k \frac{(1+|k|)^{2}}{|k|^{2}\left(1+k^{2}\right)^{2}}\right)^{1 / 2} \\
& \times\left(\int d^{3} k\left\|\left(N^{-1 / 2} a_{k}-\varphi_{t}(k)\right) U_{K} \Psi_{N, t}\right\|^{2}\right)^{1 / 2} \\
\leqq & C\left\|\psi_{t}\right\|_{H^{1}} \beta_{K}(t) .
\end{aligned}
$$

Line (6.21b). We again use (6.23) and estimate

$$
\begin{aligned}
(6.21 b) & \leqq 2 \int_{|k| \geqq K} d^{3} k|k|^{-1}\left|\left\langle\psi_{t}, e^{i k \cdot} \psi_{t}\right\rangle\right|||\left\langle U_{K} \Psi_{N, t},\left(N^{-1 / 2} a_{k}-\varphi_{t}(k)\right) U_{K} \Psi_{N, t}||\right. \\
& \leqq C\left\|\psi_{t}\right\|_{H^{1}} \int_{|k| \geqq K} d^{3} k \frac{(1+|k|)}{|k|\left(1+k^{2}\right)}\left\|\left(N^{-1 / 2} a_{k}-\varphi_{t}(k)\right) U_{K} \Psi_{N, t}\right\| \\
& \leqq C\left\|\psi_{t}\right\|_{H^{1}}\left(\beta_{K}^{b}(t)+\int_{|k| \geqq K} d^{3} k \frac{(1+|k|)^{2}}{|k|^{2}\left(1+k^{2}\right)^{2}}\right) \\
& \leqq C\left\|\psi_{t}\right\|_{H^{1}}\left(\beta_{K}^{b}(t)+K^{-1}\right) .
\end{aligned}
$$

Line (6.21c). Writing (6.21c) as

$$
2 \int_{|k| \leqq K} d^{3} k|k|^{-1} \operatorname{Im}\left\langle e^{i k x_{1}}\left(N^{-1 / 2} a_{k}-\varphi_{t}(k)\right) p_{1}^{t} U_{K} \Psi_{N, t}, q_{1}^{t} U_{K} \Psi_{N, t}\right\rangle
$$

shows that this is exactly the same expression as the second line in (6.5). We consequently have

$$
|(6.21 c)| \leqq C\left\|\psi_{t}\right\|_{H^{1}}^{2}\left(\beta_{K}^{a}(t)+\beta_{K}^{b}(t)\right) .
$$

Line (6.21d). To find a suitable bound for (6.21d) is the most difficult step in the proof. We start by estimating

$$
|(6.21 d)| \leqq 2 \int_{|k| \leqq K} d^{3} k|k|^{-1}\left|\left\langle U_{K} \Psi_{N, t}, e^{i k x_{1}} q_{1}^{t}\left(N^{-1 / 2} a_{k}-\varphi_{t}(k)\right) U_{K} \Psi_{N, t}\right\rangle\right|
$$




$$
\begin{aligned}
& =2 \int_{|k| \leqq K} d^{3} k|k|^{-1}\left|\left\langle U_{K} \Psi_{N, t}, N^{-1} \sum_{j=1}^{N} e^{i k x_{j}} q_{j}^{t}\left(N^{-1 / 2} a_{k}-\varphi_{t}(k)\right) U_{K} \Psi_{N, t}\right\rangle\right| \\
& \leqq 2 \int_{|k| \leqq K} d^{3} k|k|^{-1}\left\|N^{-1} \sum_{j=1}^{N} q_{j}^{t} e^{-i k x_{j}} U_{K} \Psi_{N, t}\right\|\left\|\left(N^{-1 / 2} a_{k}-\varphi_{t}(k)\right) U_{K} \Psi_{N, t}\right\| \\
& \leqq \beta_{K}^{b}(t)+\int_{|k| \leqq K} d^{3} k|k|^{-2}\left\|N^{-1} \sum_{j=1}^{N} q_{j}^{t} e^{-i k x_{j}} U_{K} \Psi_{N, t}\right\|^{2}
\end{aligned}
$$

The last term is bounded by

$$
\begin{aligned}
& \int_{|k| \leqq K} d^{3} k|k|^{-2}\left\|N^{-1} \sum_{j=1}^{N} q_{j}^{t} e^{-i k x_{j}} U_{K} \Psi_{N, t}\right\|^{2} \\
& \leqq 4 \pi N^{-1} K+\int_{|k| \leqq K} d^{3} k|k|^{-2}\left|\operatorname{Re}\left\langle q_{2}^{t} e^{-i k x_{2}} U_{K} \Psi_{N, t}, q_{1}^{t} e^{-i k x_{1}} U_{K} \Psi_{N, t}\right\rangle\right|
\end{aligned}
$$

With $O_{1,2}=\left(1-\Delta_{2}\right)^{-1 / 2} e^{i k x_{2}}\left(1-\Delta_{1}\right)^{1 / 2} q_{2}^{t}$ one has

$$
\begin{aligned}
e^{i k x_{2}} q_{2}^{t} q_{1}^{t} e^{-i k x_{1}}+e^{i k x_{1}} q_{1}^{t} q_{2}^{t} e^{-i k x_{2}} & =O_{2,1}^{*} O_{1,2}+O_{1,2}^{*} O_{2,1} \\
& \leqq O_{1,2}^{*} O_{1,2}+O_{2,1}^{*} O_{2,1} .
\end{aligned}
$$

Thus, using the symmetry of the wave function and $e^{-i k x_{2}}\left(1-\Delta_{2}\right)^{-1} e^{i k x_{2}}=$ $\left(\left(-i \nabla_{2}+k\right)^{2}+1\right)^{-1}$, we obtain the bound

$$
\begin{gathered}
\int_{|k| \leqq K} d^{3} k|k|^{-2}\left|\operatorname{Re}\left\langle q_{2}^{t} e^{-i k x_{2}} U_{K} \Psi_{N, t}, q_{1} e^{-i k x_{1}} U_{K} \Psi_{N, t}\right\rangle\right| \\
\leqq \int_{|k| \leqq K} d^{3} k|k|^{-2}\left\langle U_{K} \Psi_{N, t}, q_{2}^{t}\left(1-\Delta_{1}\right)^{1 / 2}\right. \\
\left.e^{-i k x_{2}}\left(1-\Delta_{2}\right)^{-1} e^{i k x_{2}}\left(1-\Delta_{1}\right)^{1 / 2} q_{2}^{t} U_{K} \Psi_{N, t}\right\rangle \\
=\left\langle\left(1-\Delta_{1}\right)^{1 / 2} q_{2}^{t} U_{K} \Psi_{N, t}, \int_{|k| \leqq K} d^{3} k\right. \\
\left.|k|^{-2}\left(\left(-i \nabla_{2}+k\right)^{2}+1\right)^{-1}\left(1-\Delta_{1}\right)^{1 / 2} q_{2}^{t} U_{K} \Psi_{N, t}\right\rangle .
\end{gathered}
$$

In combination with

$$
\begin{aligned}
& \left\|\int_{|k| \leqq K} d^{3} k|k|^{-2}\left(\left(-i \nabla_{2}+k\right)^{2}+1\right)^{-1}\right\|_{\text {op }} \\
& \quad=\sup _{p \in \mathbb{R}^{3}} \int_{|k| \leqq K} d^{3} k|k|^{-2}\left((p+k)^{2}+1\right)^{-1}<\infty
\end{aligned}
$$

(compare with (6.9)) and Lemma 5.1 this gives

$$
|(6.21 d)| \leqq C\left(\beta_{K}^{b}(t)+N^{-1} K+\left\|\left(1-\Delta_{2}\right)^{1 / 2} q_{1}^{t} U_{K} \Psi_{N, t}\right\|^{2}\right)
$$




$$
\begin{aligned}
& \leqq C\left(\beta_{K}^{a}(t)+\beta_{K}^{b}(t)+N^{-1} K+\left\|\nabla_{2} q_{1}^{t} U_{K} \Psi_{N, t}\right\|^{2}\right) \\
& \leqq C\left(\left\|\psi_{t}\right\|_{H^{2}}^{2}+\left\|\varphi_{t}\right\|_{L_{1}^{2}}^{2}+\left|E_{N}^{\mathrm{F}}\left(\Psi_{N, t}\right)\right|\right)\left(\beta_{K}(t)+N^{-1} K^{-1}+N^{-1} K\right) .
\end{aligned}
$$

Line (6.21e). We have

$$
\begin{aligned}
|(6.21 e)| \leqq & 4 \int d^{3} k|k|\left|B_{K, x}(k)\right|\left\|\left(N^{-1 / 2} a_{k}-\varphi_{t}(k)\right) U_{K} \Psi_{N, t}\right\| \\
& \times\left\|\left(i \nabla_{1}-N^{-1 / 2} \Phi\left(k B_{K, x_{1}}\right)\right) U_{K} \Psi_{N, t}\right\| \\
\leqq & C \sqrt{\beta_{K}^{b}(t)}\left\|\left(i \nabla_{1}-N^{-1 / 2} \Phi\left(k B_{K, x_{1}}\right)\right) U_{K} \Psi_{N, t}\right\|\left\||\cdot| B_{K, x}\right\| .
\end{aligned}
$$

By using (3.7), the symmetry of the wave function and (3.20), we get

$$
\begin{aligned}
|(6.21 e)| & \leqq C\left(\left\|\nabla_{1} U_{K} \Psi_{N, t}\right\|+\left\||\cdot| B_{K, x_{1}}\right\|\left\|N^{-1 / 2}(\mathcal{N}+1) U_{K} \Psi_{N, t}\right\|\right)\left(\beta_{K}^{b}(t)+K^{-1}\right) \\
& \leqq C\left(\left\langle U_{K} \Psi_{N, t},\left.N^{-1} H_{N}^{0} U_{K} \Psi_{N, t}\right|^{1 / 2}+1\right)\left(\beta_{K}^{b}(t)+K^{-1}\right)\right. \\
& \leqq C\left(\left\langle U_{K} \Psi_{N, t},\left.\left(N^{-1} H_{N, K}^{\mathrm{G}}+C\right) U_{K} \Psi_{N, t}\right|^{1 / 2}+1\right)\left(\beta_{K}^{b}(t)+K^{-1}\right) .\right.
\end{aligned}
$$

In total, we thus arrive at

$$
\left|\frac{\mathrm{d}}{\mathrm{d} t} \beta_{K}^{b}\left(\Psi_{N, t}, \varphi_{t}\right)\right| \leqq C\left(\left\|\psi_{t}\right\|_{H^{2}}^{2}+\left\|\varphi_{t}\right\|_{L_{1}^{2}}^{2}+\left|E_{N}^{\mathrm{F}}\left(\Psi_{N, t}\right)\right|\right)\left(\beta_{K}(t)+K^{-1}+\frac{K}{N}\right) .
$$

Conclusion: We combine $\frac{\mathrm{d}}{\mathrm{d} t} \beta^{c}(t)=0,(6.14)$ and (6.36) with Proposition (2.1) and $\left|E_{N}^{\mathrm{F}}\left(\Psi_{N, t}\right)\right|=\left|E_{N}^{\mathrm{F}}\left(\Psi_{N}\right)\right| \leqq E_{0}$ in order to obtain (4.15).

\section{Remaining Proofs}

\subsection{Proof of Lemma 4.1}

To show the inequality (4.1), we use $\gamma_{U_{K} \Psi_{N}}^{(1,0)}=\gamma_{U_{K, x_{1}} \Psi_{N}}^{(1,0)}$ with $U_{K, x_{1}}=$ $\exp \left(i N^{-1 / 2} \Pi\left(B_{K, x_{1}}\right)\right)$, which follows directly from (3.11) and the definition of the reduced density matrix. Hence,

$$
\operatorname{Tr}_{L^{2}\left(\mathbb{R}^{3}\right)}\left|\gamma_{\Psi_{N}}^{(1,0)}-\gamma_{U_{K} \Psi_{N}}^{(1,0)}\right| \leqq 2\left\|\left(U_{K, x_{1}}-1\right) \Psi_{N}\right\|
$$

Using $\left\|\left(U_{K, x_{1}}-1\right) \Psi_{N}\right\|=\left\|\left(U_{K, x_{1}}-1\right) U_{K} \Psi_{N}\right\|$, we obtain (4.1) from the bound

$$
\left\|\left(U_{K, x_{1}}-1\right) U_{K} \Psi_{N}\right\| \leqq 2\left\|B_{K, x}\right\|\left\|\left(\frac{\mathcal{N}+1}{N}\right)^{1 / 2} U_{K} \Psi_{N}\right\|,
$$

together with $\mathcal{N} \leqq H_{N}^{0}$, (3.20) and (3.18). Inequality (7.2) follows from the spectral calculus for self-adjoint operators, using $1-U_{K, x}=f\left(N^{-1 / 2} \Pi\left(B_{K, x}\right)\right)$ with $f(s)=1-\exp (i s)$ in combination with $|f(s)| \leqq|s|$. 
Using the properties of the Weyl operator (in particular (3.9) together with (3.11)) and

$U_{K} \mathcal{N} U_{K}^{*}=\mathcal{N}+N^{-1} \sum_{i, j=1}^{N}\left\langle B_{K, x_{i}}, B_{K, x_{j}}\right\rangle+N^{-1 / 2} \sum_{j=1}^{N}\left(a\left(B_{K, x_{j}}\right)+a^{*}\left(B_{K, x_{j}}\right)\right)$,

we have

$$
\begin{aligned}
& N^{-1}\left|\left\langle W^{*}(\sqrt{N} \varphi) \Psi_{N},\left(\mathcal{N}-U_{K}^{*} \mathcal{N} U_{K}\right) W^{*}(\sqrt{N} \varphi) \Psi_{N}\right\rangle\right| \\
& \quad=N^{-1} \mid\left\langle W^{*}(\sqrt{N} \varphi) U_{K} \Psi_{N},\left(U_{K} \mathcal{N} U_{K}^{*}-\mathcal{N}\right) W^{*}(\sqrt{N} \varphi) U_{K} \Psi_{N}\right\rangle \\
& \quad \leqq\left\|B_{K, x}\right\|^{2}+2 N^{-3 / 2} \sum_{j=1}^{N}\left\|a\left(B_{K, x_{j}}\right) W^{*}(\sqrt{N} \varphi) U_{K} \Psi_{N}\right\| \\
& \quad \leqq\left\|B_{K, x}\right\|^{2}+2\left\|B_{K, x}\right\|\|\varphi\|+2 N^{-1 / 2}\left\|B_{K, x}\right\|\left\|\mathcal{N}^{1 / 2} U_{K} \Psi_{N}\right\| .
\end{aligned}
$$

An application of (3.7) and (3.20) then leads to

$$
\begin{aligned}
(7.4) & \leqq C(1+\|\varphi\|)\left\|B_{K, x}\right\|\left(1+\left\langle U_{K} \Psi_{N}, N^{-1} H_{N}^{0} U_{K} \Psi_{N}\right\rangle^{1 / 2}\right) \\
& \leqq C K^{-3 / 2}(1+\|\varphi\|)\left\langle U_{K} \Psi_{N},\left(N^{-1} H_{N, K}^{\mathrm{G}}+C\right) U_{K} \Psi_{N}\right\rangle^{1 / 2} .
\end{aligned}
$$

In combination with (3.18), this shows (4.2).

\subsection{Proof of Proposition 2.2}

Throughout this section, we set $\xi_{N}=\psi^{\otimes N} \otimes W(\sqrt{N} \varphi) \Omega$. The bound on the energy follows from

$$
\left\langle\Psi_{N}, H_{N}^{\mathrm{F}} \Psi_{N}\right\rangle=\left\langle\xi_{N}, H_{N, K}^{\mathrm{G}} \xi_{N}\right\rangle=\left\langle\xi_{N},\left(H_{N, K}^{\mathrm{F}}+\sum_{j=1}^{N} A_{K, x_{j}}+\sum_{j, l=1}^{N} V_{K}\left(x_{j}-x_{l}\right)\right) \xi_{N}\right\rangle
$$

in combination with (3.17), (A.2) and

$$
\left|\left\langle\xi_{N}, H_{N, K}^{\mathrm{F}} \xi_{N}\right\rangle\right|=N\left|\left\langle\psi,\left(-\Delta+\Phi_{K}(\cdot, 0)\right) \psi\right\rangle+\|\varphi\|^{2}\right| \leqq C N\left(\|\psi\|_{H^{1}}^{2}+\|\varphi\|^{2}\right) .
$$

In (7.7), we used the shift property of the Weyl operators (3.10).

For the bound on $a\left(\Psi_{N}, \psi\right)$, we note that

$$
\left.\operatorname{Tr}_{L^{2}\left(\mathbb{R}^{3}\right)}\left|\gamma_{\Psi_{N}}^{(1,0)}-\right| \psi\right\rangle\left\langle\psi||=\operatorname{Tr}_{L^{2}\left(\mathbb{R}^{3}\right)}\left|\gamma_{\Psi_{N}}^{(1,0)}-\gamma_{U_{K} \Psi_{N}}^{(1,0)}\right|\right.
$$


since $|\psi\rangle\langle\psi|=\gamma_{\xi_{N}}^{(1,0)}$ and $\xi_{N}=U_{K} \Psi_{N}$. Applying Lemma (4.1), we obtain the stated estimate. The bound on $b\left(\Psi_{N}, \varphi\right)$ follows readily from (3.12),

$$
b\left(\Psi_{N}, \varphi\right)=N^{-1} \int d^{3} k\left\|a_{k} U_{K}^{*}\left(\psi^{\otimes N} \otimes \Omega\right)\right\|^{2} \leqq\left\|B_{K, x}\right\|^{2} \leqq C K^{-3} .
$$

We are thus left with the bound for $c\left(\Psi_{N}\right)$, which we write with the aid of (3.18) as

$$
c\left(\Psi_{N}\right)=\left\|N^{-1}\left(H_{N, K}^{\mathrm{G}}-\left\langle\xi_{N}, H_{N, K}^{\mathrm{G}} \xi_{N}\right\rangle\right) \xi_{N}\right\|^{2} .
$$

Recalling Definition 3.1 and using the triangle inequality, we get

$$
\begin{aligned}
\left\|\left(H_{N, K}^{\mathrm{G}}-\left\langle\xi_{N}, H_{N, K}^{\mathrm{G}} \xi_{N}\right\rangle\right) \xi_{N}\right\| & \leqq\left\|\left(H_{N, K}^{\mathrm{F}}-\left\langle\xi_{N}, H_{N, K}^{\mathrm{F}} \xi_{N}\right\rangle\right) \xi_{N}\right\| \\
& +2\left\|\sum_{j=1}^{N} A_{K, x_{j}} \xi_{N}\right\|+2\left\|\sum_{j, l=1}^{N} V_{K}\left(x_{j}-x_{l}\right) \xi_{N}\right\| .
\end{aligned}
$$

After a lengthy but straightforward computation, using the shift property (3.10) and the fact that $\Delta_{x}$ commutes with $W(\sqrt{N} \varphi)$, we find that

$$
\begin{aligned}
\frac{1}{N} & \left\|\left(H_{N, K}^{\mathrm{F}}-\left\langle\xi_{N}, H_{N, K}^{\mathrm{F}} \xi_{N}\right\rangle\right) \xi_{N}\right\|^{2} \\
& =\|\varphi\|^{2}+\left\langle\psi,(-\Delta)^{2} \psi\right\rangle-\langle\psi,(-\Delta) \psi\rangle^{2}+N^{-1}\left\langle\psi,\left\|G_{K, x}\right\|^{2} \psi\right\rangle \\
& +\left(1-N^{-1}\right)\left\|\left\langle\psi, G_{K, x} \psi\right\rangle\right\|^{2}+4\left\langle\psi,\left(\operatorname{Re}\left\langle G_{K, x}, \varphi\right\rangle\right)^{2} \psi\right\rangle-4\left\langle\psi, \operatorname{Re}\left\langle G_{K, x}, \varphi\right\rangle \psi\right\rangle^{2} \\
& +2 \operatorname{Re}\left\langle\psi,\left\langle\varphi, G_{K, x}\right\rangle \psi\right\rangle+2\left(\left\langle\psi,\left(-\Delta_{x}\right) \operatorname{Re}\left\langle G_{K, x}, \varphi\right\rangle \psi\right\rangle \text { c.c. }\right) \\
& -4\langle\psi,(-\Delta) \psi\rangle\left\langle\psi, \operatorname{Re}\left\langle G_{K, x}, \varphi\right\rangle \psi\right\rangle
\end{aligned}
$$

We shall show that the right hand side is bounded from above by a constant times $1+K N^{-1}$, with the constant depending only on $\|\psi\|_{H^{2}}$ and $\|\varphi\|_{L_{1}^{2}}$. For the first four summands (i.e., the terms in the first line), this is obvious (recall (3.7)). In the fifth summand, we can use (6.23) to conclude that

$\left\|\left\langle\psi, G_{K, x} \psi\right\rangle\right\|^{2} \leqq C$ independently of $K$. For each of the remaining terms on the right side of (7.12), we use

$$
\left\|\left\langle G_{K, x}, \varphi\right\rangle \mid \leqq\right\|(1+|\cdot|)^{-1} G_{K, x}\left\|_{2}\right\|(1+|\cdot|) \varphi \|,
$$

which is bounded by $C\|\varphi\|_{L_{1}^{2}}$. Hence, we find

$$
\left\|N^{-1}\left(H_{N, K}^{\mathrm{F}}-\left\langle\xi_{N}, H_{N, K}^{\mathrm{F}} \xi_{N}\right\rangle\right) \xi_{N}\right\|^{2} \leqq C\left(N^{-1}+K N^{-2}\right) .
$$

Next, we use

$$
\frac{1}{N}\left\|\sum_{j=1}^{N} A_{K, x_{j}} \xi_{N}\right\| \leqq\left\|A_{K, x_{1}} \xi_{N}\right\|
$$


and recalling (3.13) we estimate (with $\widetilde{\Psi}_{N}=\psi^{\otimes N} \otimes \Omega$ )

$$
\begin{aligned}
\| & N^{-1 / 2} \nabla_{1} \cdot a\left(k B_{K, x_{1}}\right) W(\sqrt{N} \varphi) \widetilde{\Psi}_{N} \| \\
& =\left\|\nabla_{1} \cdot \int d k k \overline{B_{K, x_{1}}(k)} \varphi(k) \widetilde{\Psi}_{N}\right\| \\
& \leqq\left\|\int d k k^{2} \overline{B_{K, x_{1}}(k)} \varphi(k) \widetilde{\Psi}_{N}\right\|+\left\|\int d k k \overline{B_{K, x_{1}}(k)} \varphi(k) \nabla_{1} \widetilde{\Psi}_{N}\right\| \\
& \leqq\left\||\cdot| B_{K, x}\right\|_{2}\left(\||\cdot| \varphi\|+\|\varphi\|\|\psi\|_{H^{1}}\right) \leqq C K^{-1 / 2} .
\end{aligned}
$$

Similarly, we also have

$$
\begin{aligned}
\left\|N^{-1 / 2} a^{*}\left(k B_{K, x_{1}}\right) \cdot \nabla_{1} W(\sqrt{N} \varphi) \widetilde{\Psi}_{N}\right\| & =\left\|\int d k k B_{K_{N}, x_{1}}(k)\left(N^{-1 / 2} a_{k}^{*}+\overline{\varphi(k)}\right) \cdot \nabla_{1} \widetilde{\Psi}_{N}\right\| \\
& \leqq\left\|\cdot \mid B_{K, x}\right\|\left(N^{-1 / 2}\|\psi\|_{H^{1}}+\|\varphi\|\|\nabla \psi\|\right) \leqq C K^{-1 / 2} .
\end{aligned}
$$

In order to estimate the term containing $\Phi\left(k B_{K, x}\right)^{2}$, consider

$$
W^{*}(\sqrt{N} \varphi) \Phi\left(k B_{K, x_{1}}\right) W(\sqrt{N} \varphi)=\Phi\left(k B_{K, x_{1}}\right)+2 \sqrt{N} \operatorname{Re}\left\langle k B_{K, x_{1}}, \varphi\right\rangle,
$$

and thus

$$
\begin{aligned}
& \left\|N^{-1} \Phi\left(k B_{K, x_{1}}\right)^{2} W(\sqrt{N} \varphi) \widetilde{\Psi}_{N}\right\| \\
& =N^{-1}\left\|\left(\Phi\left(k B_{K, x_{1}}\right)+2 \sqrt{N} \operatorname{Re}\left\langle k B_{K, x_{1}}, \varphi\right\rangle\right)^{2} \widetilde{\Psi}_{N}\right\| \\
& \leqq 2 N^{-1}\left\|\Phi\left(k B_{K, x_{1}}\right)^{2} \widetilde{\Psi}_{N}\right\|+8\left|\left\langle\left|k B_{K, x_{1}}\right|,|\varphi|\right\rangle\right|^{2} .
\end{aligned}
$$

In the last line, we use (3.7) to obtain

$$
\left\|\Phi\left(k B_{K, x_{1}}\right)^{2} \widetilde{\Psi}_{N}\right\|=\sqrt{3}\left\|k B_{K, x}\right\|^{2} \leqq C K^{-1} .
$$

Finally, using (3.17), we estimate

$$
N^{-1}\left\|\sum_{j, l=1}^{N} V_{K}\left(x_{j}-x_{l}\right) \xi_{N}\right\| \leqq N^{-1} \sum_{j, l=1}^{N}\left\|V_{K}\left(x_{j}-x_{l}\right) \xi_{N}\right\| \leqq C K^{-1},
$$

which completes the proof of the proposition. 


\subsection{Proof of Theorem 2.2}

Given Theorem 2.1, (2.19) follows from (4.1) together with the bound

$$
\left\|\left(1-U_{K}\right) \Psi_{N}\right\| \leqq \frac{C N}{K^{3 / 2}}\left\|\left(\frac{H_{N, K}^{\mathrm{G}}+C N}{N}\right)^{1 / 2} \Psi_{N}\right\|, \quad \Psi_{N} \in \mathcal{D}\left(H_{N}^{0}\right) .
$$

The latter follows from $\mathcal{N} \leqq H_{N}^{0}$, (3.20) and the functional calculus for self-adjoint operators, using $1-U_{K}=f\left(N^{-1 / 2} \sum_{j=1}^{N} \Pi\left(B_{K, x_{j}}\right)\right)$ with $f(s)=1-\exp (i s)$ and the bound $|f(s)| \leqq|s|$. In more detail, let $\Psi_{N, t}$ as in Theorem 2.2 and denote $\Phi_{N, t}=e^{-i H_{N}^{\mathrm{F}} t} U_{K}^{*} \Psi_{N, 0}$. Then, using (7.22),

$$
\begin{aligned}
\operatorname{Tr}_{L^{2}\left(\mathbb{R}^{3}\right)}\left|\gamma_{\Psi_{N, t}}^{(1,0)}-\gamma_{\Phi_{N, t}}^{(1,0)}\right| & \leqq 2\left\|e^{-i H_{N}^{\mathrm{F}} t}\left(\Psi_{N, 0}-\Phi_{N, 0}\right)\right\|=2\left\|\left(1-U_{K}\right) \Psi_{N, 0}\right\| \\
& \leqq \frac{C N}{K^{3 / 2}}
\end{aligned}
$$

and the triangle inequality,

$$
\left.\operatorname{Tr}_{L^{2}\left(\mathbb{R}^{3}\right)}\left|\gamma_{\Psi_{N, t}}^{(1,0)}-\right| \psi_{t}\right\rangle\left\langle\psi_{t}|| \leqq \frac{C N}{K^{3 / 2}}+\operatorname{Tr}_{L^{2}\left(\mathbb{R}^{3}\right)}\left|\gamma_{\Phi_{N, t}}^{(1,0)}-\right| \psi_{t}\right\rangle\left\langle\psi_{t}||\right.
$$

Since $\Phi_{N, 0} \in \mathcal{D}\left(H_{N}^{\mathrm{F}}\right)$ and $E_{0}=\sup _{N \in \mathbb{N}}\left|N^{-1}\left\langle\Phi_{N, 0}, H_{N}^{\mathrm{F}} \Phi_{N, 0}\right\rangle\right|<\infty$ by assumption, we infer with Theorem 2.1 that

$$
\begin{aligned}
& \left.\operatorname{Tr}_{L^{2}\left(\mathbb{R}^{3}\right)}\left|\gamma_{\Phi_{N, t}}^{(1,0)}-\right| \psi_{t}\right\rangle\left\langle\psi_{t}||\right. \\
& \quad \leqq \sqrt{a\left(\Phi_{N, 0}, \psi\right)+b\left(\Phi_{N, 0}, \varphi\right)+c\left(\Phi_{N, 0}\right)+N^{-1 / 2}} e^{C(1+|t|)^{3}} .
\end{aligned}
$$

Using Lemma 4.1, we have

$$
a\left(\Phi_{N, 0}, \psi\right) \leqq a\left(\Psi_{N, 0}, \psi\right)+C K^{-3 / 2}, \quad b\left(\Phi_{N, 0}, \varphi\right) \leqq b\left(\Psi_{N, 0}, \varphi\right)+C K^{-3 / 2}
$$

which proves the first bound in Theorem 2.2 if we set $K=K_{N} \geq c N^{5 / 6}$.

In order to prove (2.20), we estimate

$$
\begin{aligned}
& \left\langle W^{*}\left(\sqrt{N} \varphi_{t}\right) \Psi_{N, t}, \sqrt{\frac{\mathcal{N}}{N}} W^{*}\left(\sqrt{N} \varphi_{t}\right) \Psi_{N, t}\right\rangle \\
& \quad \leqq\left|\left\langle W^{*}\left(\sqrt{N} \varphi_{t}\right) \Psi_{N, t}, \sqrt{\frac{\mathcal{N}}{N}} W^{*}\left(\sqrt{N} \varphi_{t}\right) \Phi_{N, t}\right\rangle\right| \\
& \quad+\left|\left\langle W^{*}\left(\sqrt{N} \varphi_{t}\right) \Psi_{N, t}, \sqrt{\frac{\mathcal{N}}{N}} W^{*}\left(\sqrt{N} \varphi_{t}\right)\left(\Psi_{N, t}-\Phi_{N, t}\right)\right\rangle\right|
\end{aligned}
$$

with $\Phi_{N, t}$ defined as above. In the first line, we use the Cauchy-Schwarz inequality and apply Theorem 2.1 to $\Phi_{N, t}$, i.e.,

$\left\|\sqrt{\frac{\mathcal{N}}{N}} W^{*}\left(\sqrt{N} \varphi_{t}\right) \Phi_{N, t}\right\|^{2} \leqq\left(a\left(\Phi_{N, 0}, \psi\right)+b\left(\Phi_{N, 0}, \varphi\right)+c\left(\Phi_{N, 0}\right)+N^{-1 / 2}\right) e^{C(1+|t|)^{3}}$ 


$$
\leqq\left(a\left(\Psi_{N, 0}, \psi\right)+b\left(\Psi_{N, 0}, \varphi\right)+c\left(\Phi_{N, 0}\right)+K^{-3 / 2}+N^{-1 / 2}\right) e^{C(1+|t|)^{3}},
$$

where we made use of (7.26) in the second step. In (7.27b), we estimate

$$
\left\|\Psi_{N, t}-\Phi_{N, t}\right\| \leqq\left\|\left(1-U_{K}\right) \Psi_{N, 0}\right\| \leqq \frac{C N}{K^{3 / 2}}\left\|\left(\frac{H_{N, K}^{\mathrm{G}}+C N}{N}\right)^{1 / 2} \Psi_{N, 0}\right\|,
$$

together with

$$
\left\|\sqrt{\frac{\mathcal{N}}{N}} W^{*}(\sqrt{N} \varphi) \Psi_{N, t}\right\| \leqq C\left(\left\|\left(\frac{H_{N, K}^{\mathrm{G}}+C N}{N}\right)^{1 / 2} \Psi_{N, 0}\right\|+\left\|\varphi_{t}\right\|\right),
$$

which for $K=K_{N} \geqq c N^{5 / 6}$ proves (2.20). In order to show (7.30), we use the commutation relations (3.10) and $2 \Phi\left(\sqrt{N} \varphi_{t}\right) \leqq \mathcal{N}+N\left\|\varphi_{t}\right\|^{2}$, in order to find

$$
\left\langle W^{*}\left(\sqrt{N} \varphi_{t}\right) \Psi_{N, t}, \mathcal{N} W^{*}\left(\sqrt{N} \varphi_{t}\right) \Psi_{N, t}\right\rangle \leqq 2\left(\left\langle\Psi_{N, t}, \mathcal{N} \Psi_{N, t}\right\rangle+N\left\|\varphi_{t}\right\|^{2}\right) .
$$

Using (3.19) in combination with (3.20) leads to

$$
\begin{aligned}
\mathcal{N} \leqq H_{N}^{0} \leqq 2 H_{N}^{\mathrm{F}}+C N & =e^{-i H_{N}^{\mathrm{F}} t}\left(2 H_{N}^{\mathrm{F}}+C N\right) e^{i H_{N}^{\mathrm{F}} t} \\
& \leqq C e^{-i H_{N}^{\mathrm{F}} t}\left(H_{N, K}^{\mathrm{G}}+C N\right) e^{i H_{N}^{\mathrm{F}} t} .
\end{aligned}
$$

It remains to show (2.21) and (2.22): For the Pekar state $\Psi_{N}=\psi^{\otimes N} \otimes W(\sqrt{N} \varphi) \Omega$, we have

$$
a\left(\Psi_{N}, \psi\right)=0, \quad b\left(\Psi_{N}, \varphi\right)=0, \quad c\left(U_{K}^{*} \Psi_{N}\right) \leqq C\left(N^{-1}+K^{-1}+\frac{K}{N^{2}}\right),
$$

where the last bound was proven in Proposition 2.2. Thus, if we choose $K=K_{N}=$ $c N$, we obtain (2.21) and (2.22).

Acknowledgements. Financial support by the European Research Council (ERC) under the European Union's Horizon 2020 research and innovation programme (Grant Agreement No 694227; N.L and R.S.), the SNSF Eccellenza Project PCEFP2 181153 (N.L) and the Deutsche Forschungsgemeinschaft (DFG) through the Research Training Group 1838: Spectral Theory and Dynamics of Quantum Systems (D.M.) is gratefully acknowledged. N.L. gratefully acknowledges support from the NCCR SwissMAP and would like to thank Simone Rademacher and Benjamin Schlein for interesting discussions about the time-evolution of the polaron at strong coupling. D.M. thanks Marcel Griesemer and Andreas Wünsch for extensive discussions about the Fröhlich polaron.

Open Access This article is licensed under a Creative Commons Attribution 4.0 International License, which permits use, sharing, adaptation, distribution and reproduction in any medium or format, as long as you give appropriate credit to the original author(s) and the source, provide a link to the Creative Commons licence, and indicate if changes were made. The images or other third party material in this article are included in the article's Creative Commons licence, unless indicated otherwise in a credit line to the material. If material is not included in the article's Creative Commons licence and your intended use is not permitted by 
statutory regulation or exceeds the permitted use, you will need to obtain permission directly from the copyright holder. To view a copy of this licence, visit http://creativecommons.org/ licenses/by/4.0/.

Publisher's Note Springer Nature remains neutral with regard to jurisdictional claims in published maps and institutional affiliations.

\section{A. Auxiliary Bounds}

In this appendix, we collect bounds on the interaction terms of the Hamiltonians $H_{N}^{\mathrm{F}}$ and $H_{N, K}^{\mathrm{G}}$ and derive the frequently used inequalities (3.19) and (3.20). After that we comment on the proof of Lemma 3.1.

Lemma A.1. For every $\varepsilon>0, K \in(0, \infty], N \in \mathbb{N}$ and $j \in\{1, \ldots, N\}$, we have

$$
\pm N^{-1 / 2} \Phi\left(G_{K, x_{j}}\right) \leqq \varepsilon\left(-\Delta_{j}+\frac{\mathcal{N}+1}{N}\right)+2 \frac{(16 \pi)^{2}}{\varepsilon^{3}}
$$

on $L^{2}\left(\mathbb{R}^{3 N}\right) \otimes \mathcal{F}_{S}$. Moreover, with $A_{K, x}$ defined in Definition 3.1,

$$
\pm A_{K, x_{j}} \leqq \sqrt{\frac{64 \pi}{K}}\left(-\Delta_{j}+N^{-1} \mathcal{N}\right)+\frac{16 \pi}{N K}
$$

Proof. To prove (A.1), we use again the commutator method by Lieb and Yamazaki [26]. Using (3.4) and (3.7), we have

$$
\left|\left\langle\Psi_{N}, N^{-1 / 2} \Phi\left(G_{K, x_{j}}\right) \Psi_{N}\right\rangle\right| \leqq \frac{4 \pi K}{\varepsilon}\left\|\Psi_{N}\right\|^{2}+\varepsilon N^{-1}\left\langle\Psi_{N},(\mathcal{N}+1) \Psi_{N}\right\rangle,
$$

which proves (A.1) for $K \leqq 64 \pi / \varepsilon^{2}$. In the case $K>64 \pi / \varepsilon^{2}$, we write $\Phi\left(G_{K, x_{j}}\right)=$ $\Phi\left(G_{K^{\prime}, x_{j}}\right)+\left(\Phi\left(G_{K, x_{j}}\right)-\Phi\left(G_{K^{\prime}, x_{j}}\right)\right)$ with $K^{\prime}=16 \pi / \varepsilon^{2}$. For the first summand, we use (A.3) with $K$ replaced by $K^{\prime}$ and $\varepsilon$ replaced by $\varepsilon / 2$, while for the remainder, write

$$
\left\langle\Psi_{N},\left(\Phi\left(G_{K, x_{j}}\right)-\Phi\left(G_{K^{\prime}, x_{j}}\right)\right) \Psi_{N}\right\rangle=\left\langle\Psi_{N},\left[\nabla_{j}, \Phi\left(g_{x_{j}}\right)\right] \Psi_{N}\right\rangle
$$

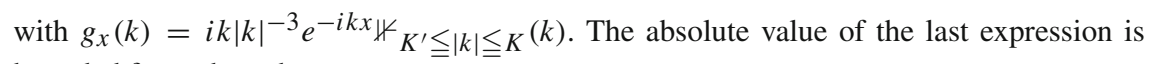
bounded from above by

$$
4\left\|\nabla_{j} \Psi_{N}\right\|\left\|g_{x}\right\|\left\|\sqrt{\mathcal{N}+1} \Psi_{N}\right\| \leqq 2 N^{1 / 2}\left\|g_{x}\right\|\left\langle\Psi_{N},\left(-\Delta_{j}+\frac{\mathcal{N}+1}{N}\right) \Psi_{N}\right\rangle .
$$

Using $\left\|g_{x}\right\| \leq \sqrt{4 \pi / K^{\prime}}=\frac{\varepsilon}{4}$ shows (A.1).

To show (A.2), we use $\left\||\cdot| B_{K, x_{j}}\right\|^{2} \leqq 4 \pi K^{-1}$ and

$$
\begin{aligned}
& \left|\left\langle\Psi_{N}, A_{K, x_{j}} \Psi_{N}\right\rangle\right| \\
& \quad \leqq 4 N^{-1 / 2}\left|\left\langle\nabla_{j} \Psi_{N}, a\left(k B_{K, x_{j}}\right) \Psi_{N}\right\rangle\right|+N^{-1}\left\|\Phi\left(k B_{K, x_{j}}\right) \Psi_{N}\right\|^{2} \\
& \leqq 4 N^{-1 / 2}\left\|\nabla_{j} \Psi_{N}\right\|\left\||\cdot| B_{K, x}\right\|\left\|\mathcal{N}^{1 / 2} \Psi_{N}\right\|+4 N^{-1}\left\||\cdot| B_{K, x}\right\|^{2}\left\|(\mathcal{N}+1)^{1 / 2} \Psi_{N}\right\|^{2} \\
& \leqq \sqrt{\frac{16 \pi}{K}}\left\langle\Psi_{N},\left(-\Delta_{j}+N^{-1} \mathcal{N}\right) \Psi_{N}\right\rangle+\frac{16 \pi}{K}\left\langle\Psi_{N}, \frac{\mathcal{N}+1}{N} \Psi_{N}\right\rangle .
\end{aligned}
$$


The previous lemma readily implies the validity of the bounds (3.19) and (3.20). With

$$
H_{N}^{\mathrm{F}}=H_{N}^{0}+N^{-1 / 2} \sum_{j=1}^{N} \Phi\left(G_{\infty, x_{j}}\right),
$$

we can use (A.1) with $\varepsilon=1 / 2$ in order to infer (3.19). Using in addition (3.17) and (A.2), one similarly obtains (3.20).

Comment on the proof of Lemma 3.1.

As already explained, Lemma 3.1 was stated and proved in [16] for the case $N=1$. Since the statement $N \geq 2$ can be proven by almost literal adaption of the argument from [16] (with obvious minor modifications), we omit all details except for the proof of the following lemma. The bound given in the lemma is one of the main ingredients in the proof, and in particular its $N$-dependence is crucial since it guarantees that we can choose $\widetilde{K}$ in Lemma 3.1 independently of $N$.

Lemma A.2. For any $\varepsilon>0$ there are $K_{\varepsilon}>0$ and $C_{\varepsilon}>0$ such that for all $N \in \mathbb{N}, K \geqq K_{\varepsilon}$ and any $\Psi_{N} \in \mathcal{D}\left(H_{N}^{0}\right)$,

$$
\left\|\left(H_{N, K}^{\mathrm{G}}-H_{N}^{0}\right) \Psi_{N}\right\| \leqq \varepsilon\left\|H_{N}^{0} \Psi_{N}\right\|+C_{\varepsilon} K N\left\|\Psi_{N}\right\| .
$$

Proof. We estimate each term in

$$
H_{N, K}^{\mathrm{G}}-H_{N}^{0}=\sum_{j=1}^{N}\left(N^{-1 / 2} \Phi\left(G_{K, x_{j}}\right)+A_{K, x_{j}}\right)+\sum_{j, l=1}^{N} V_{K}\left(x_{j}-x_{l}\right)
$$

separately. Using (3.4), $\left\|G_{K, x}\right\| \leqq C \sqrt{K}$ and $\left\|\mathcal{N}^{1 / 2} \Psi_{N}\right\|^{2} \leqq\left\|H_{N}^{0} \Psi_{N}\right\|\left\|\Psi_{N}\right\|$, we have

$$
\begin{aligned}
N^{-1 / 2} \sum_{j=1}^{N}\left\|\Phi\left(G_{K, x_{j}}\right) \Psi_{N}\right\| & \leqq C \sqrt{K N}\left(\left\|\mathcal{N}^{1 / 2} \Psi_{N}\right\|+\left\|\Psi_{N}\right\|\right) \\
& \leqq \delta\left\|H_{N}^{0} \Psi_{N}\right\|+C K N\left(1+\delta^{-1}\right)\left\|\Psi_{N}\right\|
\end{aligned}
$$

for any $\delta>0$. The norm of $\sum_{j, l=1}^{N} V_{K}\left(x_{j}-x_{l}\right) \Psi_{N}$ can be bounded using (3.17). From (3.13) we see that the remaining terms to estimate are

$$
\begin{aligned}
N^{-1} \sum_{j=1}^{N}\left\|\Phi\left(k B_{K, x_{j}}\right)^{2} \Psi_{N}\right\| & \leqq C K^{-1}\left\|(\mathcal{N}+1) \Psi_{N}\right\| \\
& \leqq C K^{-1}\left(\left\|H_{N}^{0} \Psi_{N}\right\|+\left\|\Psi_{N}\right\|\right),
\end{aligned}
$$

where we have used (3.4) and (3.7). Similarly, we have

$$
\begin{aligned}
2 N^{-1 / 2} \sum_{j=1}^{N}\left\|a^{*}\left(k B_{K, x_{j}}\right) \nabla_{j} \Psi_{N}\right\| & \leqq C \sqrt{N K^{-1}}\left\|\sqrt{\mathcal{N}+1} \nabla_{j} \Psi_{N}\right\| \\
& \leqq C K^{-1 / 2}\left(\left\|H_{N}^{0} \Psi_{N}\right\|+\left\|\Psi_{N}\right\|\right)
\end{aligned}
$$

and

$$
2 N^{-1 / 2} \sum_{j=1}^{N}\left\|\nabla_{j} a\left(k B_{K, x_{j}}\right) \Psi_{N}\right\| \leqq 2 N^{-1 / 2} \sum_{j=1}^{N}\left(\left\|a\left(k B_{K, x_{j}}\right) \nabla_{j} \Psi_{N}\right\|+\left\|a\left(|k|^{2} B_{K, x_{j}}\right) \Psi_{N}\right\|\right)
$$




$$
\leqq C K^{-1 / 2}\left\|H_{N}^{0} \Psi_{N}\right\|+2 N^{1 / 2}\left\|a\left(|k|^{2} B_{K, x_{1}}\right) \Psi_{N}\right\|
$$

In order to estimate the second summand, we use

$$
a^{*}\left(|k|^{2} B_{K, x_{1}}\right) a\left(|k|^{2} B_{K, x_{1}}\right) \leqq \widetilde{C}_{K}\left(1-\Delta_{1}\right) \mathcal{N},
$$

where

$$
\widetilde{C}_{K}=\sup _{h \in \mathbb{R}^{3}} \int_{|k| \geqq K} d^{3} k \frac{1}{\left(1+|k|^{2}\right)\left(1+(h-k)^{2}\right)} .
$$

The bound (A.14) is analogous to (6.8) and can be proven in the same way as [10, Lemma 10] (see also [15, Lemma B.5]). If one estimates the integral in (A.15) using the Cauchy-Schwarz inequality one sees that $\widetilde{C}_{K} \rightarrow 0$ for $K \rightarrow \infty$. We thus have

$$
\begin{aligned}
2 N^{1 / 2}\left\|a\left(|k|^{2} B_{K, x_{j}}\right) \Psi_{N}\right\| & \leqq 2 \widetilde{C}_{K} N^{1 / 2}\left\|\left(1-\Delta_{j}\right)^{1 / 2} \mathcal{N}^{1 / 2} \Psi_{N}\right\| \\
& =2 \widetilde{C}_{K}\left\langle\Psi_{N}, \mathcal{N} \sum_{j=1}^{N}\left(1-\Delta_{j}\right) \Psi_{N}\right\rangle^{1 / 2} \\
& \leqq \widetilde{C}_{K}\left(\left\|H_{N}^{0} \Psi_{N}\right\|+N\left\|\Psi_{N}\right\|\right),
\end{aligned}
$$

and hence,

$$
2 N^{-1 / 2} \sum_{j=1}^{N}\left\|\nabla_{j} a\left(k B_{K, x_{j}}\right) \Psi_{N}\right\| \leqq\left(\widetilde{C}_{K}+C K^{-1 / 2}\right)\left(\left\|H_{N}^{0} \Psi_{N}\right\|+N\left\|\Psi_{N}\right\|\right) .
$$

Choosing $K$ large enough and $\delta$ sufficiently small completes the proof of the lemma.

\section{B. Time Derivative of $\beta_{K}^{b}(t)$}

Because of the unboundedness of the annihilation operator, it is not directly obvious that one can use the product rule of differentiation to obtain (6.15a) and (6.15b). Its rigorous justification relies on the estimate (for $\chi_{N} \in \mathcal{D}\left(H_{N}^{0}\right)$ )

$$
\left\|\mathcal{N} \chi_{N}\right\| \leqq\left\|H_{N}^{0} \chi_{N}\right\| \leqq 2\left\|H_{N, K}^{\mathrm{G}} \chi_{N}\right\|+C K N\left\|\chi_{N}\right\|
$$

which follows from Lemma A.2. Since $U_{K} \Psi_{N, t}=e^{-i H_{N, K}^{\mathrm{G}} t} U_{K} \Psi_{N, 0}$, this together with the strong continuity of $e^{-i H_{N, K}^{\mathrm{G}} t}$ implies

$$
\lim _{h \rightarrow 0}\left\|(\mathcal{N}+1) U_{K}\left(\Psi_{N, t+h}-\Psi_{N, t}\right)\right\|=0 \text { for all } U_{K} \Psi_{N, 0} \in \mathcal{D}\left(H_{N}^{0}\right) .
$$

Note that

$$
\begin{aligned}
\beta_{K}^{b}\left(\Psi_{N, t+h}, \varphi_{t+h}\right)-\beta_{K}^{b}\left(\Psi_{N, t}, \varphi_{t}\right)= & \beta_{K}^{b}\left(\Psi_{N, t+h}, \varphi_{t}\right)-\beta_{K}^{b}\left(\Psi_{N, t}, \varphi_{t}\right) \\
& +\beta_{K}^{b}\left(\Psi_{N, t+h}, \varphi_{t+h}\right)-\beta_{K}^{b}\left(\Psi_{N, t+h}, \varphi_{t}\right)
\end{aligned}
$$


and that the first line is given by

$$
\begin{aligned}
(B .3 a)= & 2 N^{-1} \operatorname{Re}\left\langle W\left(\sqrt{N} \varphi_{t}\right) \mathcal{N} W^{*}\left(\sqrt{N} \varphi_{t}\right) U_{K} \Psi_{N, t}, U_{K}\left(\Psi_{N, t+h}-\Psi_{N, t}\right)\right\rangle \\
& +N^{-1}\left\langle W\left(\sqrt{N} \varphi_{t}\right) \mathcal{N} W^{*}\left(\sqrt{N} \varphi_{t}\right) U_{K}\left(\Psi_{N, t+h}-\Psi_{N, t}\right), U_{K}\left(\Psi_{N, t+h}-\Psi_{N, t}\right)\right\rangle .
\end{aligned}
$$

In the second line we use that

$$
\begin{aligned}
& \left\|\mathcal{N} W^{*}\left(\sqrt{N} \varphi_{t}\right) U_{K}\left(\Psi_{N, t+h}-\Psi_{N, t}\right)\right\| \\
& \quad \leq C\left(1+N\left\|\varphi_{t}\right\|^{2}\right)\left\|(\mathcal{N}+1) U_{K}\left(\Psi_{N, t+h}-\Psi_{N, t}\right)\right\| \rightarrow 0
\end{aligned}
$$

as $h \rightarrow 0$ because of (B.2) and obtain

$$
\begin{aligned}
& \lim _{h \rightarrow 0} h^{-1}\left(\beta_{K}^{b}\left(\Psi_{N, t+h}, \varphi_{t}\right)-\beta_{K}^{b}\left(\Psi_{N, t}, \varphi_{t}\right)\right) \\
& \quad=-2 N^{-1} \operatorname{Re}\left\langle W\left(\sqrt{N} \varphi_{t}\right) \mathcal{N} W^{*}\left(\sqrt{N} \varphi_{t}\right) U_{K} \Psi_{N, t}, i H_{N, K}^{\mathrm{G}} U_{K} \Psi_{N, t}\right\rangle
\end{aligned}
$$

with Stone's theorem. Next, we consider

$$
\begin{aligned}
(B .3 b)= & -2 \operatorname{Re} \int d^{3} k\left\langle\left(\varphi_{t+h}(k)-\varphi_{t}(k)\right) U_{K} \Psi_{N, t+h},\left(N^{-1 / 2} a_{k}-\varphi_{t}(k)\right) U_{K} \Psi_{N, t+h}\right\rangle \\
& +2 \operatorname{Re} \int d^{3} k\left\langle\left(\varphi_{t+h}(k)-\varphi_{t}(k)\right) U_{K} \Psi_{N, t},\left(N^{-1 / 2} a_{k}-\varphi_{t}(k)\right) U_{K} \Psi_{N, t}\right\rangle \\
& +\left\|\varphi_{t+h}-\varphi_{t}\right\|^{2} \\
& -2 \operatorname{Re} \int d^{3} k\left\langle\left(\varphi_{t+h}(k)-\varphi_{t}(k)\right) U_{K} \Psi_{N, t},\left(N^{-1 / 2} a_{k}-\varphi_{t}(k)\right) U_{K} \Psi_{N, t}\right\rangle .
\end{aligned}
$$

Using the Cauchy-Schwarz inequality we estimate the first two terms by

$$
\begin{aligned}
& |(B .7 a)+(B .7 b)| \\
& \leqq \\
& \quad 2 \int d^{3} k\left|\varphi_{t+h}(k)-\varphi_{t}(k)\right|\left(\left|\left\langle U_{K} \Psi_{N, t+h},\left(N^{-1 / 2}-\varphi_{t}(k)\right) U_{K}\left(\Psi_{N, t+h}-\Psi_{N, t}\right)\right\rangle\right|\right. \\
& \left.\quad+\left|\left\langle U_{K}\left(\Psi_{N, t+h}-\Psi_{N, t}\right),\left(N^{-1 / 2} a_{k}-\varphi_{t}(K)\right) U_{K} \Psi_{N, t}\right\rangle\right|\right) \\
& \leqq \\
& \quad 2 N^{-1 / 2}\left\|\left(\varphi_{t+h}-\varphi_{t}\right)\right\|\left(\left\|\mathcal{N}^{1 / 2} W^{*}\left(\sqrt{N} \varphi_{t}\right) U_{K}\left(\Psi_{N, t+h}-\Psi_{N, t}\right)\right\|\right. \\
& \left.\quad+\left\|U_{K}\left(\Psi_{N, t+h}-\Psi_{N, t}\right)\right\|\left\|\mathcal{N}^{1 / 2} W^{*}\left(\sqrt{N} \varphi_{t}\right) U_{K} \Psi_{N, t}\right\|\right) .
\end{aligned}
$$

Stone's theorem and (B.5) then lead to

$$
\begin{aligned}
& \lim _{h \rightarrow 0} h^{-1}\left(\beta^{b}\left(\Psi_{N, t+h}, \varphi_{t+h}\right)-\beta_{K}^{b}\left(\Psi_{N, t+h}, \varphi_{t}\right)\right) \\
& =-2 \operatorname{Re} \int d^{3} k\left\langle\left(\partial_{t} \varphi_{t}(k)\right) U_{K} \Psi_{N, t},\left(N^{-1 / 2} a_{k}-\varphi_{t}(k)\right) U_{K} \Psi_{N, t}\right\rangle .
\end{aligned}
$$

In combination, this shows (6.15a) and (6.15b). 


\section{References}

1. Ammari, Z., Falconi, M.: Bohr's correspondence principle for the renormalized Nelson model. SIAM J. Math. Anal. 49(6), 5031-5095, 2017

2. Benedikter, N., de Oliviera, G., Schlein, B.: Quantitative derivation of the GrossPitaevskii equation. Commun. Pure Appl. Math. 68(8), 1399-1482, 2015

3. Brennecke, C., Schlein, B.: Gross-Pitaevskii dynamics for Bose-Einstein condensates. Anal. PDE 12(6), 1513-1596, 2019

4. Carlone, R., Correggi, M., Falconi, M., Olivieri, M.: Microscopic Derivation of Time-dependent Point Interactions. Preprint, arXiv:1904.11012 2019.

5. Correggi, M., Falconi, M., Olivieri, M.: Quasi-Classical Dynamics. Preprint, arXiv:1909.13313 2019.

6. Davies, E.B.: Particle-boson interactions and the weak coupling limit. J. Math. Phys. 20, 345-351, 1979

7. Falconi, M.: Classical limit of the Nelson model with cutoff. J. Math. Phys. 54(1), 012303, 2013

8. Frank, R.L., Gang, Z.: Derivation of an effective evolution equation for a strongly coupled polaron. Anal. PDE 10(2), 379-422, 2017

9. Frank, R.L., GANG, Z.: A non-linear adiabatic theorem for the one-dimensional Landau-Pekar equations. J. Funct. Anal. 279(7), 108631, 2020

10. Frank, R.L., Schlein, B.: Dynamics of a strongly coupled polaron. Lett. Math. Phys. 104, 911-929, 2014

11. Frank, R.L., Seiringer, R.: Quantum corrections to the Pekar asymptotics of a strongly coupled polaron. Commun. Pure Appl. Math. 74(3), 544-588, 2021

12. Ginibre, J., Nironi, F., Velo, G.: Partially classical limit of the Nelson model. Ann. H. Poincaré 7, 21-43, 2006

13. Ginibre, J., Velo, G.: The classical field limit of scattering theory for nonrelativistic many-boson systems I. Commun. Math. Phys. 66(1), 37-76, 1979

14. Ginibre, J., Velo, G.: The classical field limit of scattering theory for nonrelativistic many-boson systems II. Commun. Math. Phys. 68(1), 45-68, 1979

15. Griesemer, M.: On the dynamics of polarons in the strong-coupling limit. Rev. Math. Phys. 29(10), 1750030, 2017

16. Griesemer, M., WÜNSCH, A.: Self-adjointness and domain of the Fröhlich Hamiltonian. J. Math. Phys. 57(10), 021902, 2016

17. Gross, E.P.: Particle-like solutions in field theory. Ann. Phys. 19, 219-233, 1962

18. Hepp, K.: The classical limit for quantum mechanical correlation functions. Commun. Math. Phys. 35, 265-277, 1974

19. Hiroshima, F.: Weak coupling limit with a removal of an ultraviolet cutoff for a Hamiltonian of particles interacting with a massive scalar field. Infin. Dimens. Anal. Quantum 1, 407-423, 1998

20. Jeblick, M., LeOpold, N., Pickl, P.: Derivation of the time dependent Gross-Pitaevskii equation in two dimensions. Commun. Math. Phys. 372, 1-69, 2019

21. Landau, L.D., Pekar, S.I.: Effective mass of a polaron. Zh. Eksp. Teor. Fiz. 18(5), 419-423, 1948

22. Leopold, N., Petrat, S.: Mean-field dynamics for the Nelson model with Fermions. Ann. H. Poincaré 20(10), 3471-3508, 2019

23. Leopold, N., Pickl, P.: Derivation of the Maxwell-Schrödinger equations from the Pauli-Fierz Hamiltonian. SIAM J. Math. Anal. 52(5), 4900-4936, 2020

24. LEOPOLD, N., PICKL, P.: Mean-field limits of particles in interaction with quantized radiation fields. (Eds. D. Cadamuro, M. Duell, W. Dybalski and S. Simonella) Macroscopic Limits of Quantum Systems, Volume 270 of Springer Proceedings in Mathematics and Statistics, 185-214 2018.

25. Leopold, N., Rademacher, S., Schlein, B., Seiringer, R.: The Landau-Pekar equations: Adiabatic theorem and accuracy. Preprint, arXiv:1904.12532 2019, Anal. \& PDE (in press). 
26. Lieb, E.H., YamaZaki, K.: Ground-state energy and effective mass of the polaron. Phys. Rev. 111, 728-733, 1958

27. Mitrouskas, D., Petrat, S., Pickl, P.: Bogoliubov corrections and trace norm convergence for the Hartree dynamics. Rev. Math. Phys. 31(8), 1950024, 2019

28. NELSON, E.: Interaction of nonrelativistic particles with a quantized scalar field. J. Math. Phys. 5(9), 1190-1197, 1964

29. PICKL, P.: A simple derivation of mean field limits for quantum systems. Lett. Math. Phys. 97, 151-164, 2011

30. PiCKL, P.: Derivation of the time dependent Gross-Pitaevskii equation with external fields. Rev. Math. Phys. 27(1), 1550003, 45, 2015

31. Rodnianski, I., Schlein, B.: Quantum fluctuations and rate of convergence towards mean field dynamics. Commun. Math. Phys. 291(1), 31-61, 2009

32. Teufel, S.: Effective $N$-body dynamics for the massless Nelson model and adiabatic decoupling without spectral gap. Ann. H. Poincaré 3, 939-965, 2002

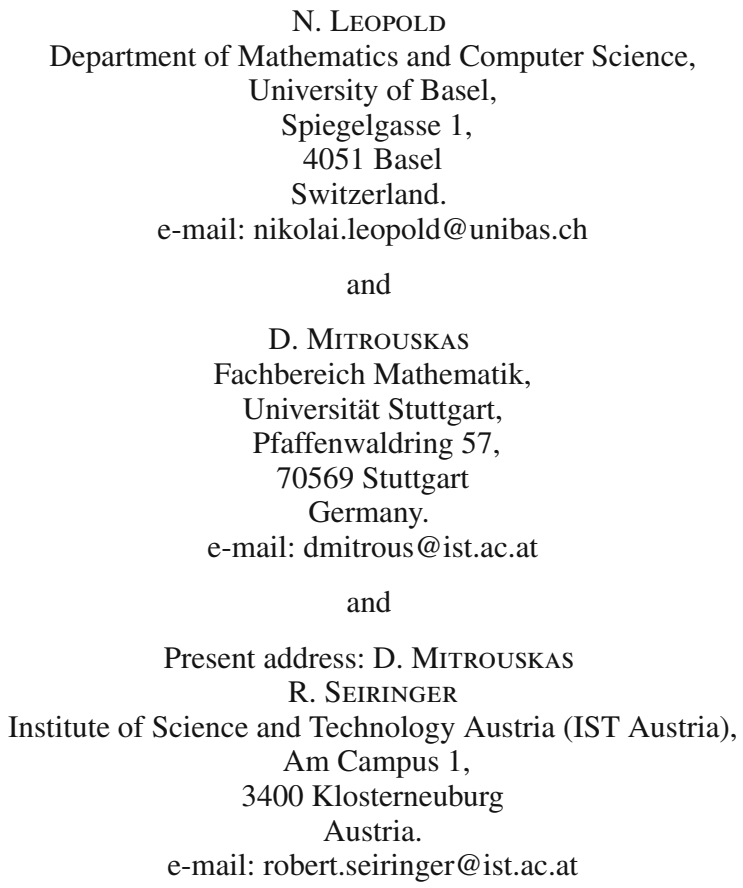

(Received February 10, 2020 / Accepted January 30, 2021)

Published online February 26, 2021

(C) The Author(s) (2021) 